\title{
Réflexions sur les mesures ponctuelles du coefficient de perméabilité par essais Lefranc
}

\section{CASSAN}

Directeur technique honoraire de Fondasol 6. me du Petit-Limas 84000 Avignon
Nous présentons et commentons, dans cette communication, une mêthode d'interprétation des essais Lefranc qui permet de mettre en évidence les diverses anomalies susceptibles de se produire dans le déroulement des essais et d"en fausser les résultats : colmatage dans les essais par injection, décolmatage ou entrainements d'éléments fins ctans les essais par pompage.

Cette méthode consiste à étudier d'une part l'évolution, en fonction du temps et sous débit constant, de la charge hydraulique dans la cavité de filtration, et d'autre part l'évolution de la vìtesse relative de l'eau dans le tube d'écoulement reliant cette cavité ả la surface du sol. En ajustant une courbe theorique sur les points expérimentaux, il est alors possible de déterminer une valeur representative du coefficient de perméabilité. même si cet ajustement n'est que partiel à condition qu'il ne concerne qu'tun ensemble de points consécutifs à partir du début de l'essai.

Cette méthode a élé illustrée par quelques exemples de courbes, mais aussi par des résultats obtenus sur deux grands chantiers où l'on a pu montrer une honne concordance des résultats d'une part entre les essais Lefranc par injection et ceux par pompage mais également entre les essais Lefranc et les essais de pompage classiques dans un puits de gros diamètre assortis de piézomètres satellites.

Mots-clés : cavité de filltration, tube d'écoulement, charge hydraulique différentielle, charge variable, charge constante, injection, prélèvement, pompage, débil, vitesse relative d'écoulement, graphe des vitesses, colmatage, décolmatage, anomalies, courbe théorique, ajustement.
NDLA : Les discussions sur cet article sont acceptées jusqu'au fir awril 2005.

\section{Reflections on the ponctual measures of the coefficient of permeability in Lefranc's tests}

A method of interpretation of Lefranc's tests is presented and commerted. The paper points out the possible abnornal occurrences that can affect the result: sealing in the injection tests, in unsallng or washing away of fine particles in pumping tests

The rnethod consists of studying the change in the hydratic load under a constant flow in the cavity of filtration in relation wht time, and the change of the weter relative speed in the draining pipe linking the cavity to the ground surface. $B y$ acjusting a theoretical curve to tests data, it is possible to determine a figure representative of the permeability coefficient, even if this adjustment is only partial, provided it includes a set of consecutive points at the beginning of the test. 
The method is illustrated by some examples of curves and by results from two big construction sites. The results show a great similarity between the Lefranc's tests by injection and by pumping, and between the Lefunc's tests and classic pumping tests in a large well fitted with surrounding piezomeiers.

K\&y words: cavity of filtration, differential, al hydraulic load. variable load, constant load. injection. extraction, pumping. Flow, relative speed of drainage, graph of speed, sealing. unsealing, anomalies, theoretical curve, adjustment.

La détermination du coefficient de perméabilité des sols est quelque chose de très délicat et de très incertain qui peut se faire en laboratoire certes, avec toutes les rêserves que cela implique, mais qui s'opère le plus généralement in situ, soit par des essais ponctuels, plus précisément des essais Lefranc, soit par des essais do pompage avec mesures dans des piézomètres satelites.

Si l'unanimité se fait autour de l'essai de pompage, il n'en est pas de même de l'essaj Lefranc et il faut bien reconnaître que certains arguments avancés par ses détracteurs ne sont pas sans fondements. Pourtant, malgré les controverses quil l'entourent encore, cet essai est de plus en plus utilisé, vraisemblablement à cause de sa simplicité qui n'est d'ailleurs qu'apparente. C'est pourquoi, il convient de faire le point sur son efficacité et sa fiabilitè à la lumiére de l'expérience acquise depuis une vingtaine d’années tant dans le domaine expérimental que dans celui de l'interprétation.

\section{Principe et réalisation de l'essai}

Rappelons que l'essai Lefranc consiste d'une part à provoguer la percolation de l'eau à travers la paroj d'une cavité aménagée à l'intérieur d’un massif aquifére en créant, dans certe cavité, une charge hydraulique différentielle li par rapport à la charge hydrostatique initiale, et d'autre part à mesurer l'évolution de cette charge différentielle en fonction du temps.

Le premiar problème qui se pose donc est celui de la réalisation de cette cavité car ćest d'elle que dépen- dent, en grande partie, la qualité et la représentativité de l'essai. Je ne fais gu'évoquer ce problème car les procédures de réalisation de cette cavité ont été parfaitement décrites dans la norme NFP 94.132 après de longues et laborieuses discussions.

A lout instant l'écoulement doit satisfaire à l'écua tion ce continuité qui, en milieu homogène et isotrope, et en négligeant la compressibilité de l'eau et du sol, est whe équation de Laplace dont l'intégration conduit ả la relation fondamentale :

$$
Q_{\varepsilon}=m k h B
$$

Dans laquelie :

- Q est le débit qui traverse la paroi de la cavité

-B est le diamètre efficace de la cavité que l'on assimilo au diamètre du forage au fond duquel celle-ci a été aménagée:

- mest le coefficient de forme de la cavite dont l'expression analytique est fonction de son élancement, c'està-dire du rapport $\lambda$ entre sa longueur et son diamètre efficace.

Les expressions analytiques de m sont données dans le tableau ci-après (Cassan, 2004) et figurent dans la norme NFP.94.132;

-k est le coefficient de perméabilité du milieu supposé homogene et isotrope.

La charge hydraulique différentielle peut être produite en injectant ou en prélevant, dans la cavité, un débit constant Q (Fig. 1), et c'est autour de ce choix entre prélèvement et injection que se développe essen. tiellement la controverse.

TAalEaU I Expressions analytiques du coefficient de forme.

\begin{tabular}{|c|c|}
\hline$\lambda$ & $m$ \\
\hline$\lambda \geq 10$ & $\frac{2 \pi \lambda}{\ln 2 \lambda}$ \\
\hline $1,2 \leq \lambda<10$ & $\frac{2 \pi \lambda}{\ln \left(\lambda+\sqrt{\left.\lambda^{2}+1\right)}\right.}$ \\
\hline $0,7 \leq \lambda<1,2$ & $\pi \sqrt{4 \lambda+1}$ \\
\hline $0,3 \leq \lambda<0,7$ & $\frac{\pi}{\sqrt{2} \sqrt{4 \lambda+1}}$ \\
\hline $0<\lambda<0,3$ & $\frac{\pi \sqrt{1-4 \lambda^{2}}}{2 \operatorname{Arccotg}\left(2 \lambda+\sqrt{\left.4 \lambda^{2}+1\right)}\right.}$ \\
\hline 0 & 2 \\
\hline
\end{tabular}


L'expérience montre, en effet, que la méthode par injection conduit souvent à des phénomènes de colmatage qui peuvent être, soit ur colmatage direct des parois de la cavité, soit la formation d'une zone de colmatage qui se crée à l'extérieur de la cavitẻ, au sein même du massif, par déplacement et concentration des éléments les plus fins du sol sous l'action des forces de courant dirigêes, dans ce cas, vers l'intérieun du massif. Comme l'essal par injection est beaucoup plus facile à rẻaliser que l'essai par prélèvement, lequel se fạit en général par pompage ce qui nécessite des forages de plus gros diamètre. il tend, à l'heure actuelle, à se généraliser, de telle sorte que l'on ne dispose que de peu d'éléments de comparaison entre les deux méthodes, et que l'expérience que l'on a sur la méthode par pompage r.est pas très riche.

La méthode par prélêvement recommandée par la norme, présente une première difficulté qui est celle de la stabilité de la paroí de la cavité pendant l'essai.

On croit avoir résolu le problème en utilisant un manchon crépiné type manchon Lefranc, mais cette solution est illusoire car le manchon crépiné ne met pas à l'abri d'un entraînement des matériaux les plus fins du sol qui peuvent pénétrer dans la cavité sil les trous de la crépine sont trop importants, ce qui rend alors l'essai, sinon inexploitable, tout au moins peu représentatif par suite de l’augmentation de la porosité du sol sur une zone concentrique à la cavité

Lorscue celle-ci est constituée par un tube crépiné que l'on descend dans un forage prêalable, il faut êviter de l'entourer d'un géotextile qui est un élément favorable au colmatage externe. Mais le filtre en gravillon que l'on interpose entre la tube crépiné et la paroi du forage (Fig. 2) peut lui aussi se colmater.

Par ailleurs, dans les terrains finement granulaires, le phénomène de renard avec remontée du sol dans le tubage, est extrêmement fréquent, beaucoup plus fréquent que ne peuvent l'être les phénomènes de colmatage dans les essais par injection.

Avant de procéder à l'essai, il faudrait développer ia cavité, mais si le développement est efficace, c'est-àdire s'il n'y a pas de colmatage au cours du développement, la perméabilité de la zone développée devient plus importante que celle du sol non perturbé et on ne maîtrise plus le diamètre efficace de la cavité, car il n'est plus alors question d'assimiler ce diamètre à celuj de son forage. (a)

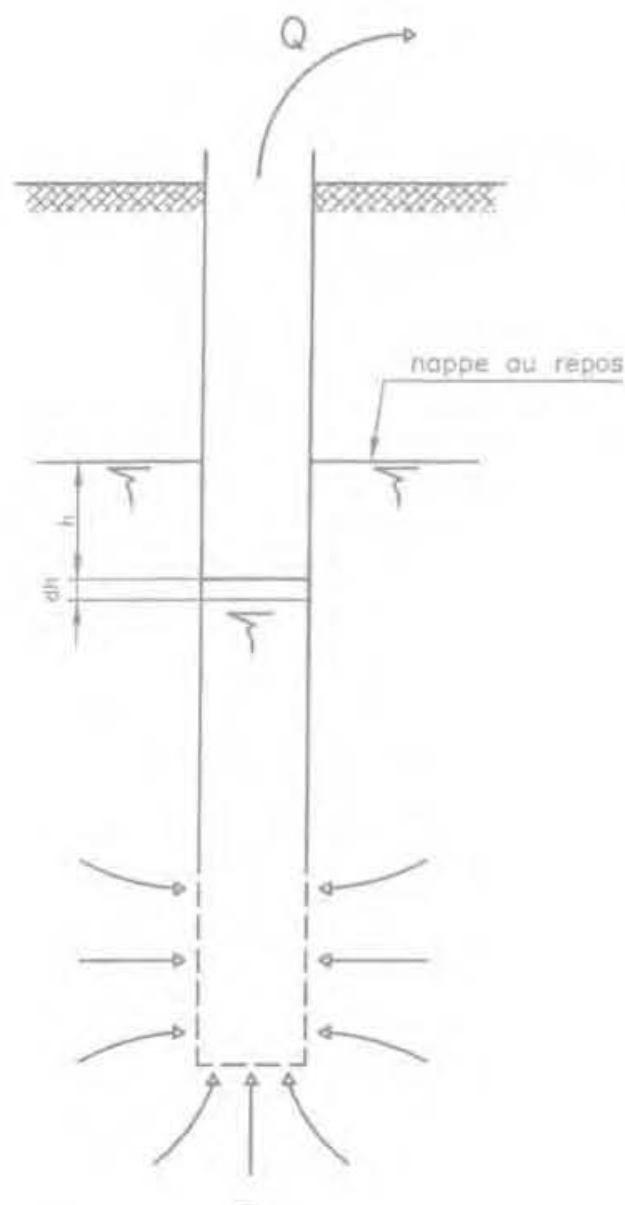

Qs (b)

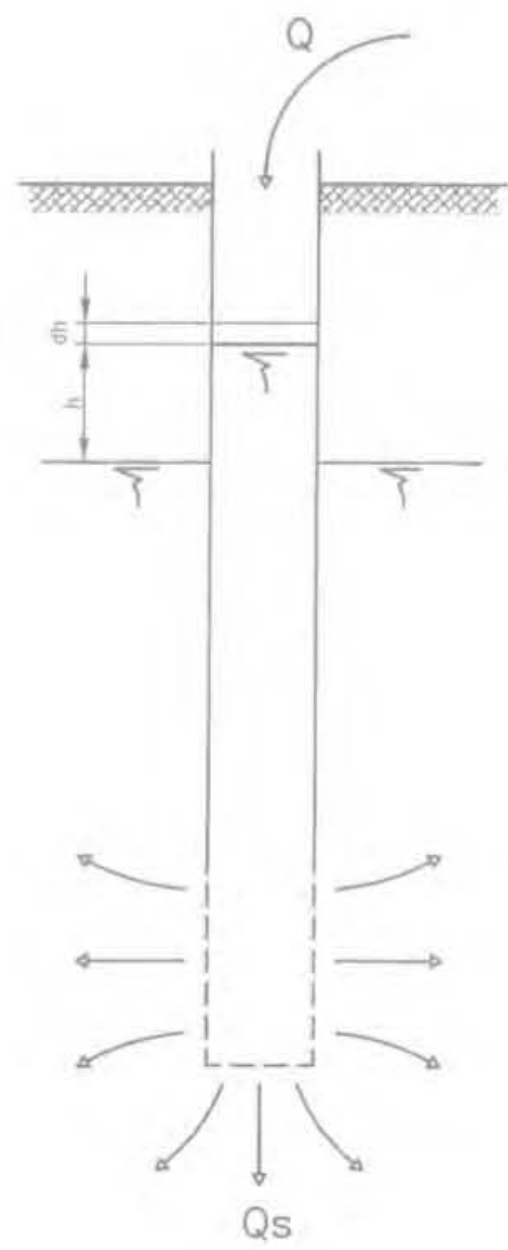

AG. 1 Principe de l'essai Lefranc.

a) par prélévement

b) par injection 


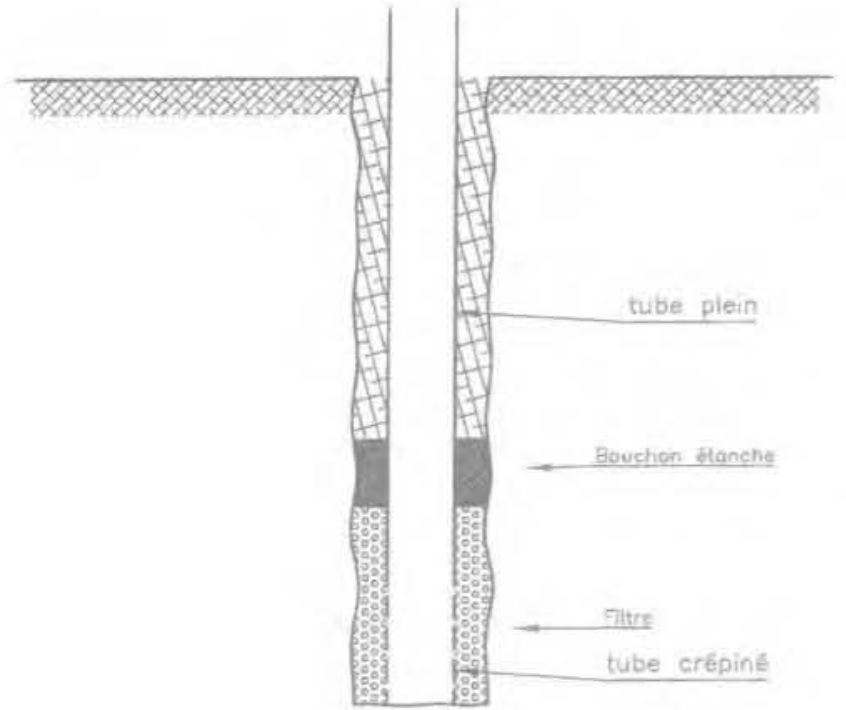

月G. Cavité constituée par un tube crépiné
Dans le cas d'un décolmatage ou d'ur renard on a $k \leq k^{*}<\infty$, et l'on voit alors que pour $k^{*}=\infty$ on a $\frac{\mathrm{k}_{\mathrm{a}}}{\mathrm{k}}=\frac{\mathrm{R}}{\mathrm{r}}$. Ainsi donc lorsque la perturbation est maximale, le coefficient de perméabilité apparent tend vers une valeur limite finie.

Si par exemple, $\frac{R}{r}=2$ on aura toujours:

$$
k \leq k \leq 2 k
$$

ce quil n'est pas grave.

En revanche un colmatage, qui correspond à $0 \leq \mathrm{k}^{*} \leq \mathrm{k}$, conduit à une êvaluation de $\mathrm{k}_{\mathrm{z}}$ beaucoup plus éloignée de la réalité et par conséquent beaucoup plus dangereuse, comme le montre le tableau II ci-dessous correspondant à $\frac{R}{r} 1,2$ et $\frac{R}{r}=2$

\section{TAR:ABII II}

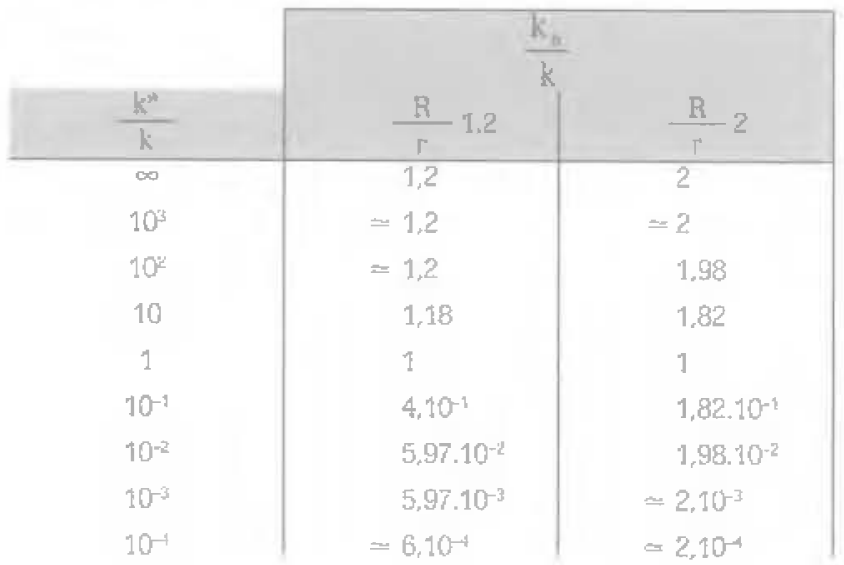

On voit donc que les deux méthodes, par pompage et par injection, présentent l'une et l'autre de nombreux inconvếnients et que jeter un interdit sur" la méthode par injection conduirait a la disparition progressive de l'essai Lefranc, ce quî serait regrettable car, comme nous le verrons par la suite, les méthodes récentes d'interprétation permettent d'apprécier la représentativité de chaque essai ef de s'affranchir en grande partie des perturbations évoquées précédemment, en particulier de celles réstutant de colmatages qui sont beat. coup plus dangereuses que celles provenant d'ur décolmatage ou d'un simple renard, comme on peut s'en rendre compte, ne serait-ce que qualitativement. en se plaçant dans le cas théorique très simple d'une cavité sphírique.

Supposons en effet que l'essai Lefranc produise une perturbation hydraulique qui se développe depuis la paroi de la cavité sphéríque de rayon r jusqu'à la sphère concentrique de rayon $\mathrm{R}$, et que cette perturbation se traduise, sur toute l'épaisseur $\mathrm{R}$ - I de la zone perturbée, par un coefficient de perméabilité $k^{*}$ constant mais différent du coefficient de perméabilité k initial du sol. Dans ces conditions, l'essai Lefranc conduira à un coefficient de perméabilité apparent $k$. différent de $\mathrm{k}^{\star}$ et de $\mathrm{k}$.

Il est alors facile de démontrer (Cassan, 2004] que :

$$
\frac{k_{\mathrm{a}}}{\mathrm{k}}=\frac{\frac{\mathrm{R}}{\mathrm{r}}}{1+\frac{\mathrm{R}}{\mathrm{r}}-1}
$$

On entend souvent parier d'essai Lefranc à charge constante et d'essai Lefranc ả charge variable. II convient de bannir du langage celte dichotomie qui provient d'une ancienne pratique que j'ai d'ailleurs utilisée moi-même au dêbut de ma cartềre mals que j’ai condamnée et abandonne depuis longtemps, et qui est schématisée sur la figure 3 . Cette pratique consiste à injecter un débit $Q_{1}$ dans la cavité par l'internédiaire $\mathrm{d}^{*} \mathrm{u} n$ tube muni d'une surverse qui permet d'évacuer. dans un bac gradué, le débit excédentaire $Q_{2}$. Le débit $\mathrm{Q}_{5}$ absorbé par le sol est alors:

La charge hydraulique differentielle $h$, qui correspond a ce débit, n'est autre que la distance verticale nappe. Elle est par conséquent constante.

L'essaỉ lui-même consistait alors à mesurèr, en fonction du temps, l'abaissement du niveau de l'eau dans le bac, ce qui permettait de calculer le volume cumulé $\mathrm{V}$, de l'eau injecté dans le sol au temps t

$$
V_{s}(t)=\int_{0}^{t}\left(Q_{1}-Q_{2}\right) d t
$$

Lorsque tout se passait bien, $Q_{2}$ était constant et la courbe $V_{s}$ (t) était une droite passant par l'origine. Le débit $Q_{\mathrm{s}}=\frac{\mathrm{dV}_{\mathrm{s}}}{\mathrm{dt}}$ qui pérétrait dans le sol était donc

$$
\mathrm{Q}_{\mathrm{s}}=\mathrm{Q}_{1}-\mathrm{Q}_{2}
$$
entre la surverse et le niveau statique initial de la 


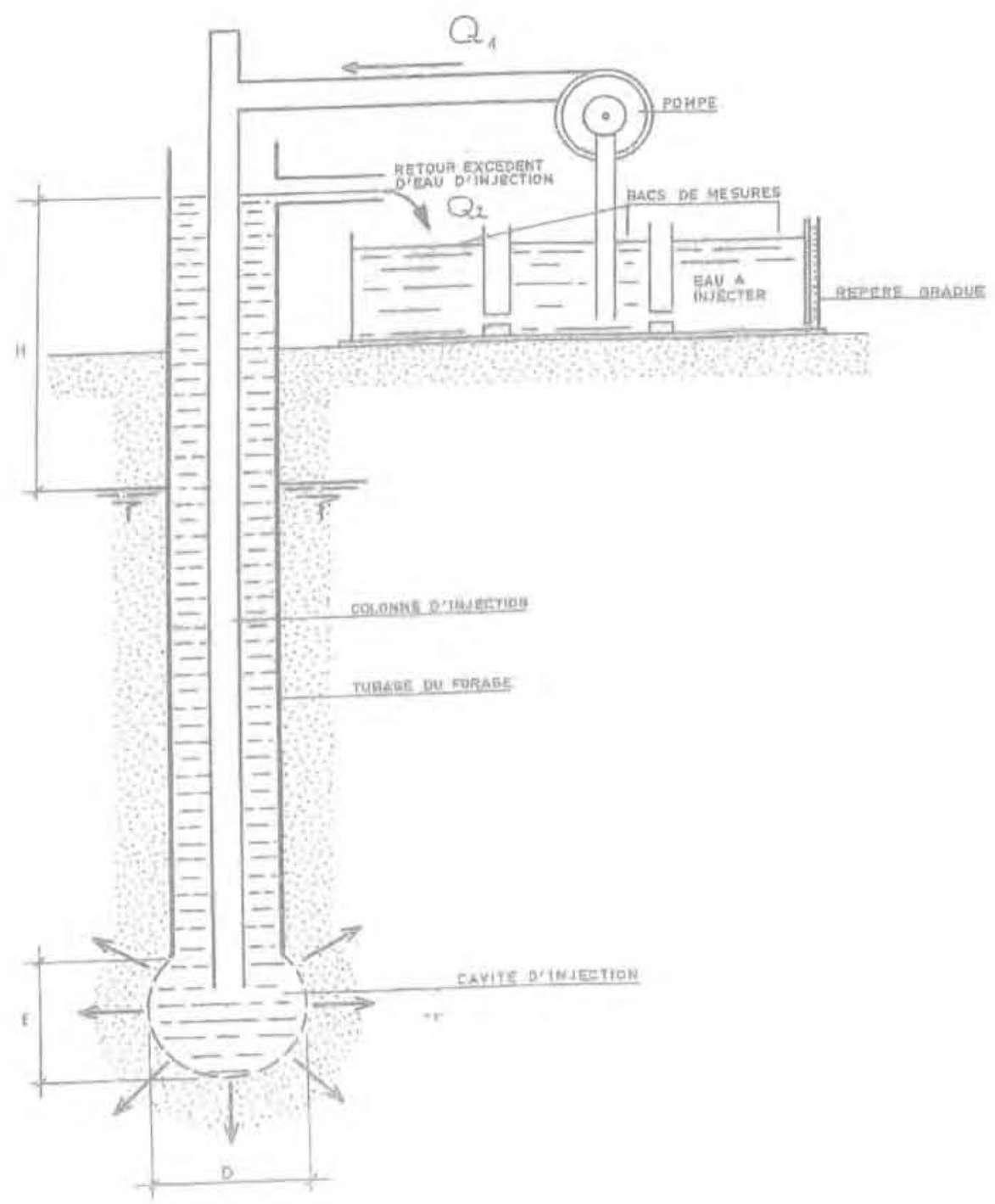

Essał Lefranc par injection ả charge constante.

constant et comme la charge différentielle était également constante, on avait donc, avec cette méthode :

$$
\mathrm{k}=\frac{\mathrm{Q}_{\mathrm{s}}}{\mathrm{mhB}}
$$

Mais lorsque la courbe $V_{s}(t)$ n'était plus une droite, on se trouvait bien embarrassé, sauf si la courbe passait sans ambiguité par l'origine, auquuel cas on pouvait achmettre comme débit la pente de la tangente íl'origine de cette courbe.

D'une façon générale jorsqu'on injecte ou que l'on pompe un débit constant dans une cavité sans imposer une valeur de la charge différentielle, cette charge évoIue en fonction du temps et ne devient constante que lorsque le débit qui traverse la paroj de la cavité est devenu égal au débit imposé : c’est le régime permanent dans lequel l'essai devient un essai à charge constante. Mais, comme nous le verrons plus loin, ce régine n'est pas toujours atteint.

C'est pourquoi la procéclure de l'essai, telle gu'elle est d'ailleurs décrite dans la norme, consiste ã injecter ou à prélever directement un débit constant Q dans la cavité et à relever, en fonction du temps, le niveau de l'eau dans le tube d'écoulement qui relie la cavité aux dispositifs de surface. On peut alors calculer les charges hydrauliques qui sont égales, à tout instant, à la différence entre le niveau de l'eau dans le tube d'écoulement et le niveau statique initial de la nappe au repos.

Ge niveau de la nappe au repos est un paramètre particulièrement important car il a une grande influence sur la valeur de $\mathrm{k}$, mais il est d'autant plus difficile à appréhender que le sol est moins perméable. compte tenu du temps de réponse des forages.

I n’est arrivé d"entendre dire que ce n'est pas le nivea de la nappe au repos qu'll convenait de prendre en compte, mais le niveau de l'eau dans le sol a l'extérieur de la cavité, mais à son voisinage jmmédiat. Indépendamment du fait que cette mesure nécessiterait des dispositions tout à fait particulières et très difficiles à mettre en ouvre, elle ne servirait à rien, car son utilisation serait incorrecte sur le plan théorique.

En effet, dans l'intégration de l'écuation de Laplace, les conditions aux limites sont les valeurs de la charge hydraulique respectivement dans la cavité et à l'infini.

Soit donc (Fig. 4) :

$-z_{r}$ la cote d'un point quelconque situé à l intérieur de la cavité: 
- h. la hauteur d'eau dans le tube d'écoulement au-dessus de ce point:

- u la pression hydraulique en ce point:

$-h_{p}$ la hauteur initiale du niveau de la nappe au repos (niveau hydrostatique) au-dessus du plan horizonta! passant par ce point (cote $\left.z_{c}\right)$ :

- $\mathrm{u}_{0}$ la pression hydraulique à l’infini à la cote $z_{c}$;

$-y_{w}$ le poids volumique de l'eau.

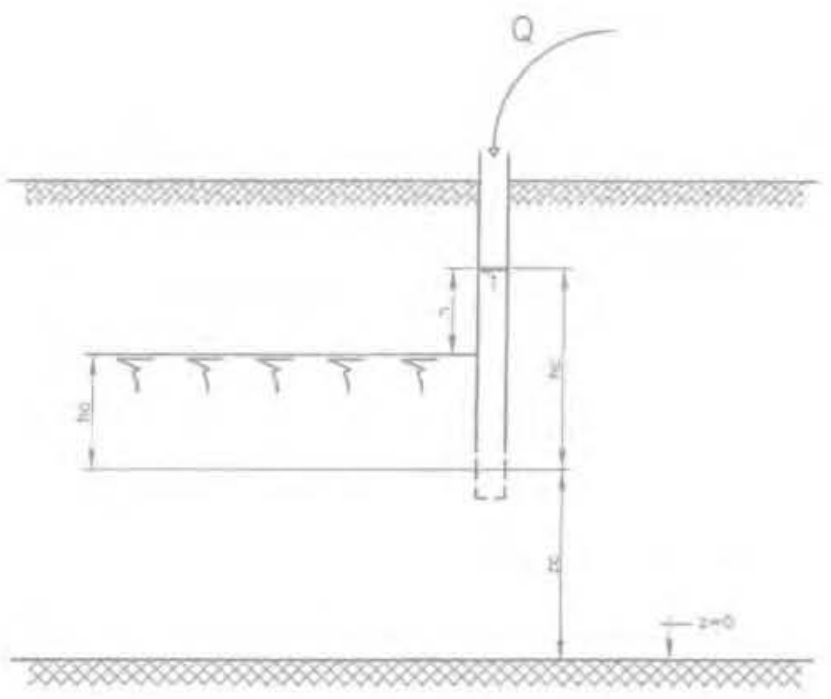

FG. Conditions aux limites dans un essai par injection.

La charge hydrauifue dans la cavité est alons. d'après l'équation de Bernouilli:

$$
\varphi_{c}=z_{c}+\frac{u_{c}}{\gamma_{s}}=z_{c}+h_{c}
$$

La charge hydraulique à l’infini est de même :

$$
\Phi_{0}=z_{c}+\frac{\omega_{0}}{\gamma_{w}}=z_{\tau_{i}}+h_{0}
$$

La différence de charge (ou difference de potentiel) $\Delta \varphi=\varphi_{-}-\varphi_{\text {r }}$ qui engendre le molvement de l'eau à travers la parof de la cavité, est donc:

$$
\varphi_{\mathrm{c}}-\varphi_{0}=\mathrm{h}_{\mathrm{r}}-\mathrm{h}_{0}=\mathrm{h}
$$

Elle est donc constante et la cavité est bien une surface equipotentielle.

On voit bien que le niveau initial de la nappe représente l'une des conditions aux limites du probleme. Tout se passe alors comme si la cavité était soumise à un potentiel égal à h sur sa paroi et nul à l'infinil.

A partir des mesures du niveau de l'eau dans te tube d'écoltement de la cavité, mesures manualles ou de préférence mesures enregistrées automatiquement, on peut alors tracer, раг points, la courbe de l'évolution de la charge hydraulique différentielle en fonction du temps (Fig. 5).

\section{Interprétation de l'essai}

\section{1}

\section{Méthode de la courbe théorique}

L'essai est interprété à partir de la courbe de la figure 5 , l"ensemble des couples de coorconnées [h, th mesurées constituant les données du problème. On voit apparaître sur cette courbe deux domaines, l'un, le domaine 1 correspondant à un régime transitcire au cours duquel la charge évolue en fonction du temps et l'autre, domaine 2, correspondant à un régime permanent dans lequel la charge reste constante.

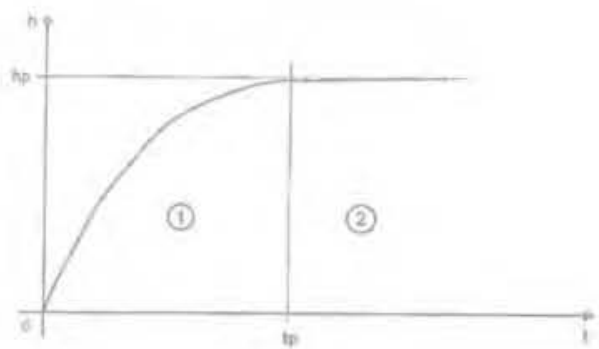

FG. 5 Évolution de la charge différentielle en fonction du temps.

(1) régime transitoire

(2) régime transitoire
Or la relation (1). qui relie le débit $Q$, percolant dans le sol à la charge $h$. est valable quel que soit h et par conséquent à tout instert.

Supposons alors qu'on réalise un essai en prélevant un débit $Q$ constant dans la cavité.

A l'instant $t$, la cavité est alimentée par un débil $\mathrm{Q}$, provenant de la nappe. Pendant un temps dt, le volume d'eau prélevé dans la cavité est Qdt et le volume d'alimentation est $Q_{\text {g }} \mathrm{dt}$.

L'abaissement du niveau de l'eau dans le tube signtfie donc que le volume de prélèvement est supérieur au volume d'alimentation. La différence entre ces deux volumes est donc égale au volume correspondant à l'abaissement dh de l'eau dans le tube d'écoulement pendant le temps dt.

Si 5 désigne l'aire de la section droite intértieure de ce tube, on obtient donc:

$$
\mathrm{Qdt}-\mathrm{Q}_{\mathrm{B}} \mathrm{dt}=\mathrm{S} \mathrm{dh}
$$

Soit :

$$
Q-Q_{s}=S \frac{d h}{d t}
$$

De même, si on opère par injection d'un débit $Q$ le débit de remontée de l'eau dans le tube d’écoulement est alors égal à la différence entre le débit qui alinente la cavité et le débit $Q_{s}$ qui en sort pour s'écouler dans le sol. 
On a donc la même relation, entre les débits, que précédemment et on peut écrire dans les deux cas:

$$
\mathrm{S} \frac{\mathrm{dh}}{\mathrm{dt}}+\mathrm{Q}_{\mathrm{s}}=\mathrm{Q}
$$

soit encore puisque : $Q_{s}=m k B h$

$$
\mathrm{S} \frac{\mathrm{dh}}{\mathrm{dt}}+\mathrm{mkBh}=\mathrm{Q}
$$

La séparation des variables est évidente et l'on a immédiatement, entre les instants $t_{0}$ et $t$ :

$$
\int_{L_{\pi}}^{t} d t=\int_{l_{0}}^{h} \frac{S d h}{Q-m k B h}
$$

soit :

$$
h=\frac{Q}{m k B}+\left(h_{0}-\frac{Q}{m k B}\right) \exp \left[-\frac{m k B}{S}\left(t-t_{0}\right)\right]
$$

Si $h_{0}=0$ et $t_{0}=0$, lexpression précédente se simplifie et devient:

$$
\mathrm{h}=\frac{\mathrm{Q}}{\mathrm{mkB}}\left[1-\exp \left(-\frac{\mathrm{mkB}}{\mathrm{S}} \mathrm{t}\right)\right]
$$

Dans les deux cas, le graphe de h présente donc une asymptote horizontale d'ordonnée $\frac{\mathrm{Q}}{\mathrm{mkB}}$ qui correspond par conșécuent au régime permanent.

Dans le cas simple de la forme (5), on voit que:

$$
\frac{\mathrm{dh}}{\mathrm{dt}}=\frac{\mathrm{Q}}{\mathrm{S}} \exp \left(-\frac{\mathrm{mkB}}{\mathrm{S}} \mathrm{t}\right)
$$

et que, par conséquent, la pente de la tangente à l'ori gine de la courbe h ( $\mathrm{t}$ ) est égale a $\frac{\mathrm{Q}}{\mathrm{S}}$.

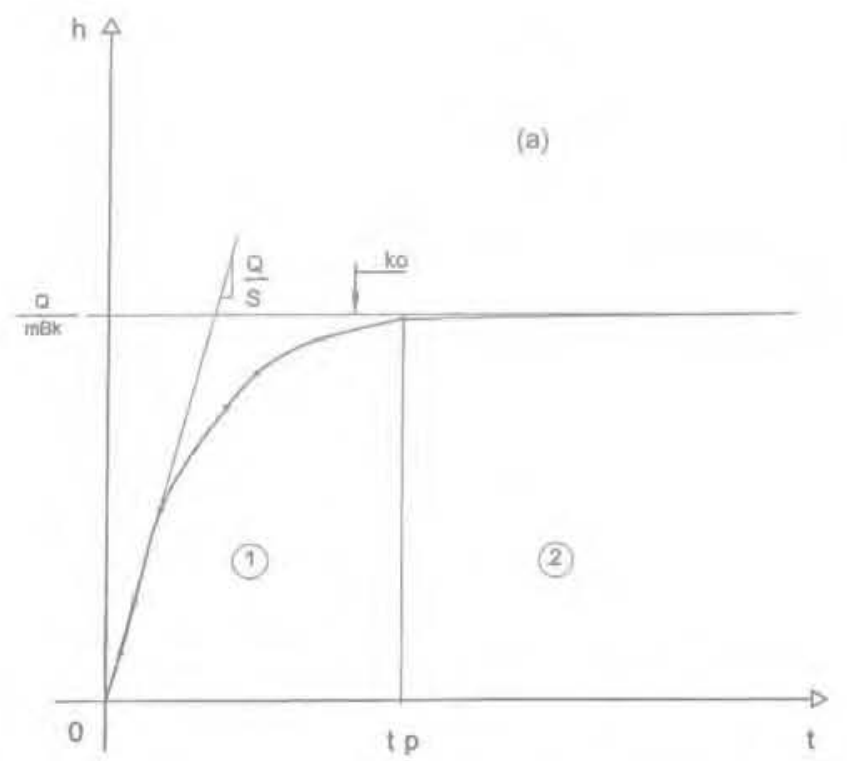

Toutes les courbes théoriques dofvent donc être tangentes à cette droite.

L'interprétation de l'essai se fait alors à l'aide d'un logiciel qui permet de tracer la courbe theorique ci-dessus lorsqu'on connait k (Fig. 6a).

On introduit donc dans le micro-ordinateur les couples de valeurs expérimentales (h, t), dont les points représentatifs apparaissent sur l'écran et, en se fjxant différentes valeurs de k, on cherche une courbe théorique quí s'ajuste le mieux possible sur les points expérimentaux. Cette méthode de superposition, qui est courante dans les sciences de la Terre en particulier pour linterprétation des essais de pompage, comme dans les méthodes de Theìs (1940) en milieu homogène, de Hantush (1955 et 1964) dans le cas de la drainance, ou de Boulton (1954 et 1963) dans le cas de débit retardé. savèe particulierement simple et rapide dans le cas présent, car elle ne demande que deux ou trois minutes par essai après la saisie des données.

Lorsque l'ajustement n'est pas possible par suite d'une anomalie de l'essai, on cherche une courbe cui s'ajuste sur les premiers points du graphe et la valeur de k correspondante est lia valeur cherchée.

Les principaux cas d'anomalies quj petvent se pré senter sont schématisés sur la figure 7 .

\section{2}

\section{Méthodes de la courbe des vitesses relatives}

On dérive la courbe de charge expérimentale de la figure 5 en calculant la pente $\frac{\Delta h}{\Delta t}$ de la tangente en différents points de cette courbe. Or $\frac{\Delta h}{\Delta t}$ r'est autre que la vitesse relative $\mathrm{v}$ du mouvement de l'eau dans le tube d'écoulement par rapport à Ta vitesse ce percolation

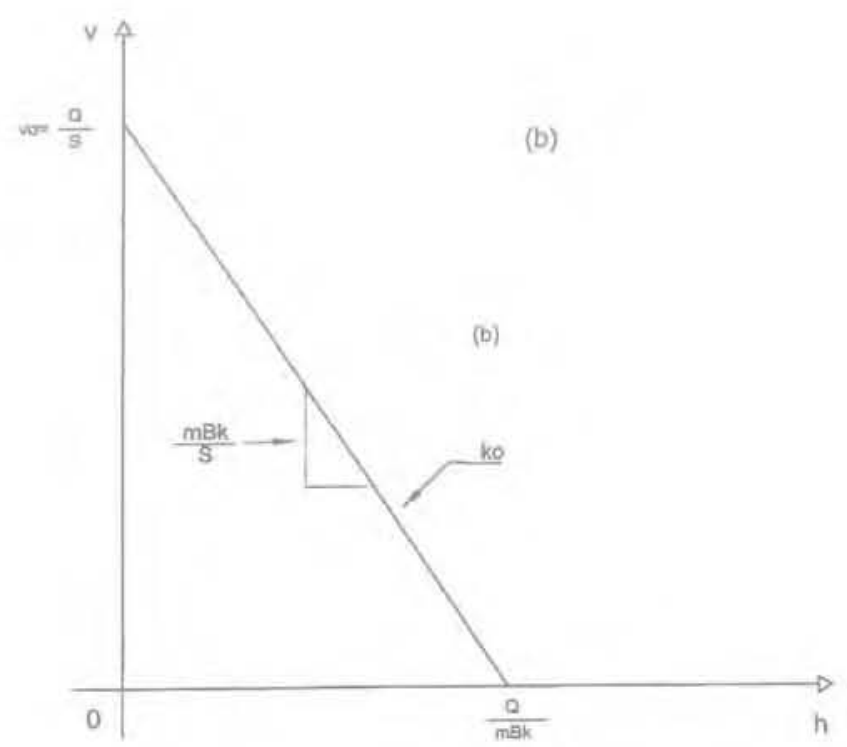

HG.6 Évolution théorique de la charge différentielle en fonction du temps (a) et de la vitesse dans le tube d'écoulement en fonction de la charge (b). 

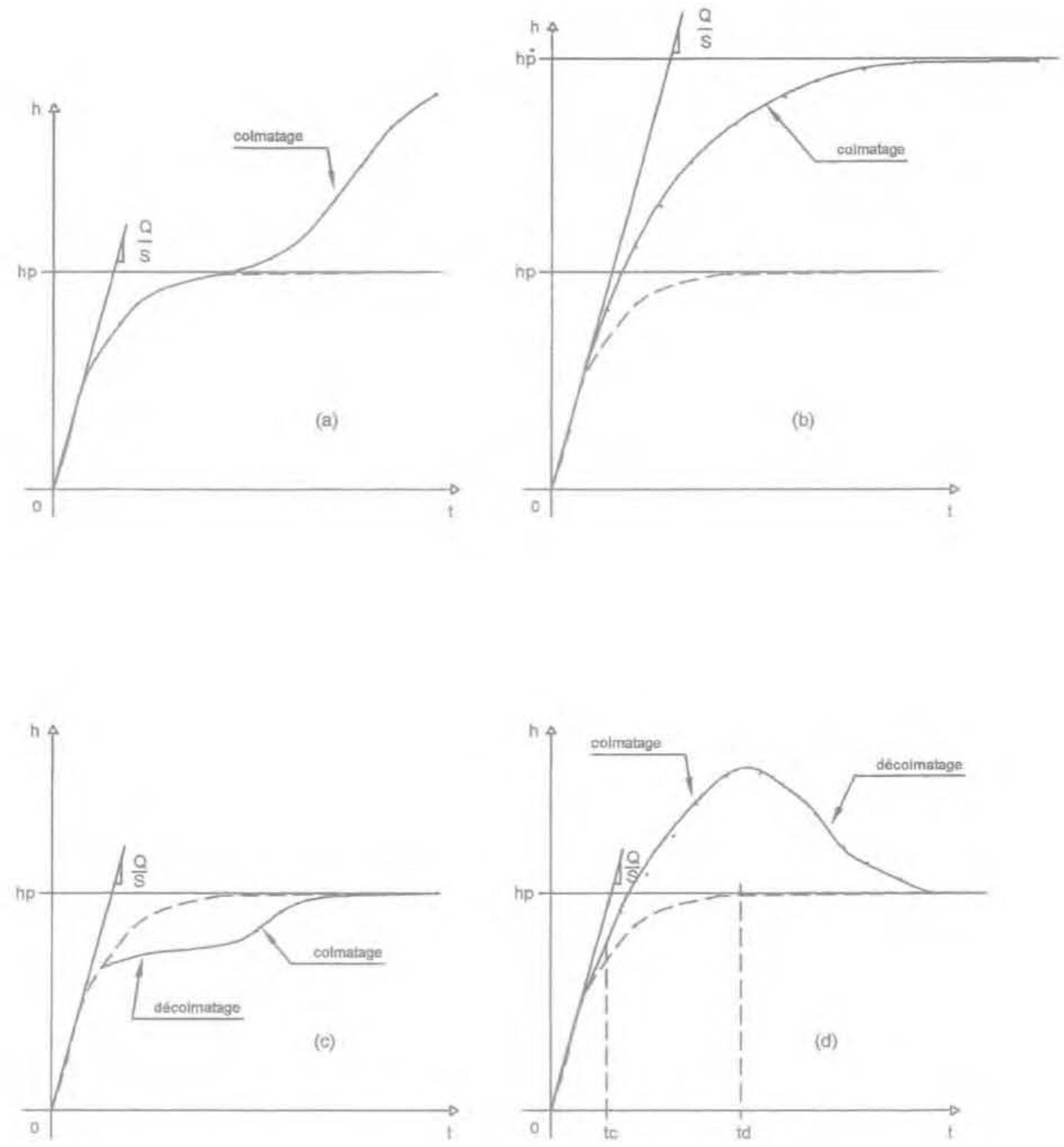

ma. 1 Principaux cas d'anomalies de l'évolution de la charge en fonction du temps. — Courbe expérimentale.

- - Courbe théorique s'ajustant sur les premiers poinfs expérimentaux.

à travers la paroi de la cavité comme on peut s'en rendre compte d'après l'équation $(2)$.

On trace alors le graphe de $v=\frac{\Delta h}{\Delta t}$ en fonction de

h. En vertu de l'équation différentielle (3), ce graphe doit être une droite de pente $-\frac{m k B}{\mathrm{~S}}$, et dont l'abscisse à l’origine est égale à $\frac{\mathrm{Q}}{\mathrm{mkb}}$, et l'ordonnée à l'origine à $\frac{\mathrm{Q}}{\mathrm{S}}$ (Fig. 6b). On dispose donc de deux évaluations de $\mathrm{k}$.
Cette ordonnée à l’origine est particulièrement importante car elle constitue une donnée incontournable du problème, puisque Q est le débit que l'on injecte ou que l'or pompe dans le tube d'écoulement et S est la surface de la section draite de ce tube. Ce sont donc deux grandeurs parfaitement connues et parfaitement constantes. Le rapport $\frac{Q}{S}$ n'est autre que la vitesse moyenne de circulation de l'eau dans une conduite qui véhicule, à pleine section, le débit Q imposé. 
Puisque la droite v (h) doit obligatoirement passer par le point d'abscisse nulle et d'ordonnée $\frac{Q}{S}$, il en résulte que toute configuration dans laquelle l'ordonnée à l’origine de la droite expérimentale est différente de $\frac{\mathrm{Q}}{\mathrm{S}}$ traduit une anomalje.

Les anomalies les plus fréquemment rencontrées sont données sur les figures 8 et 9 ou les aroites expérimentales sont figurées en traits pleins et leurs prolongements, jusqu'aux axes de coordonnées, en trait discontinu. sés

Nous allons analyser brièvement les cinq cas expo-

Le graphe expérmental de v (h) [Fig. Ba) est constitué de delux segments de droite $\mathrm{AB}$ et $\mathrm{BC}$. Le point $\mathrm{A}$, situé sur l’axe vertical a pour ordonnée $\frac{\mathrm{Q}}{\mathrm{S}}$. Le segment $\mathrm{AB}$ correspond donc à la réponse hydraulique d'un sol non perturbé et sa pente permet de calculer le coefficient de perméabilite k représentatif de ce sol. L'abscisse du point $\mathrm{A}^{\prime}$. intersection du prolongement de $\mathrm{AB}$ avec l'axe des charges correspond au régime permanent qu'gn aurait obtenu en l'absence de perturbation.

Le segment BC correspond à la réponse hydraulique d'un sol perturbé. Comme la pente de BC est plus faible que celle de $A B$, le coefficient de perrréabilité $\mathrm{k}^{*}$ est plus faible que $k$. L'abscisse $h^{*}$ du point $C$ correspond au rêgime permanent réllement obtenu, quj est relatif at terrain colmaté. On a donc $\quad k^{*}=\frac{Q}{m b h_{p}^{*}}<k$
puisque $h_{p}^{*}>h_{p^{*}}$
La droite complète C'BC correspondrait à un essai non perturbé dans un sol homogène de coefficient de perméabilité $k^{*}$ dans lequel le débit de pompage fou d'injection) serait $\mathrm{Q}^{*}<\mathrm{Q}$. Dans la représentation h (t) ce cas correspond à celui illustré par la figure $7 \mathrm{~b}$.

Sur la figure $8 \mathrm{~b}$, le graphe des vitesses relatives est également constitué de deux segments de droite, mais alors que dans la figure $8 a$ le sommet $\mathrm{B}$ du triangla ABC était situé du côté de l'orighe des coordonnées par rapport à la base $\mathrm{AC}$, dans le cas présent le sommet $B$ est situé de l'autre côté de AC.

En raisonnant comme précédemment, on voit que le segment $A B$ est relatif au sol non perturbé et permet d'évaluer le coefficient de perméabilité $\mathrm{k}$ représentatif de ce sol et que la perturbation représentée par le segment $\mathrm{BC}$ correspond a un coefficient de perméabilité $k^{* *}>k$. La droite complète CBC" représenterait un essai dans un sol non perturbé de coefficient de perméabilité $\mathrm{k}^{\text {* }}$ dans lequel on aurait pompé ou injecté un débit $\mathrm{Q}^{* * *}>\mathrm{Q}$. La perturbation est donc un decolmatage ou, ce quil revient au mêmé, un débourrage.

Sur la figure 8c, le graphe des vitesses présente une première phase linéaire d'ordonnée à I'origine $\frac{\mathrm{Q}}{\mathrm{S}}$, puis la vitesse devient constante et le graphe est constitué par une droite parallèle à l'axe des charges. Il est facile de montrer que cette deuxième phase corresoond à un colmatage progressif au cours duquel le produit kh reste constant.

Posons, en effet, pour simplifier l'écriture $\frac{\mathrm{Q}}{\mathrm{S}}=\mathrm{v}_{0}$, et soif $\mathrm{v}$ la valeur constante de la vitesse de l'eau dans le tube d'ecoulement.

L'équation (3) peut alors s'écrire dans cette deuxiême phase :
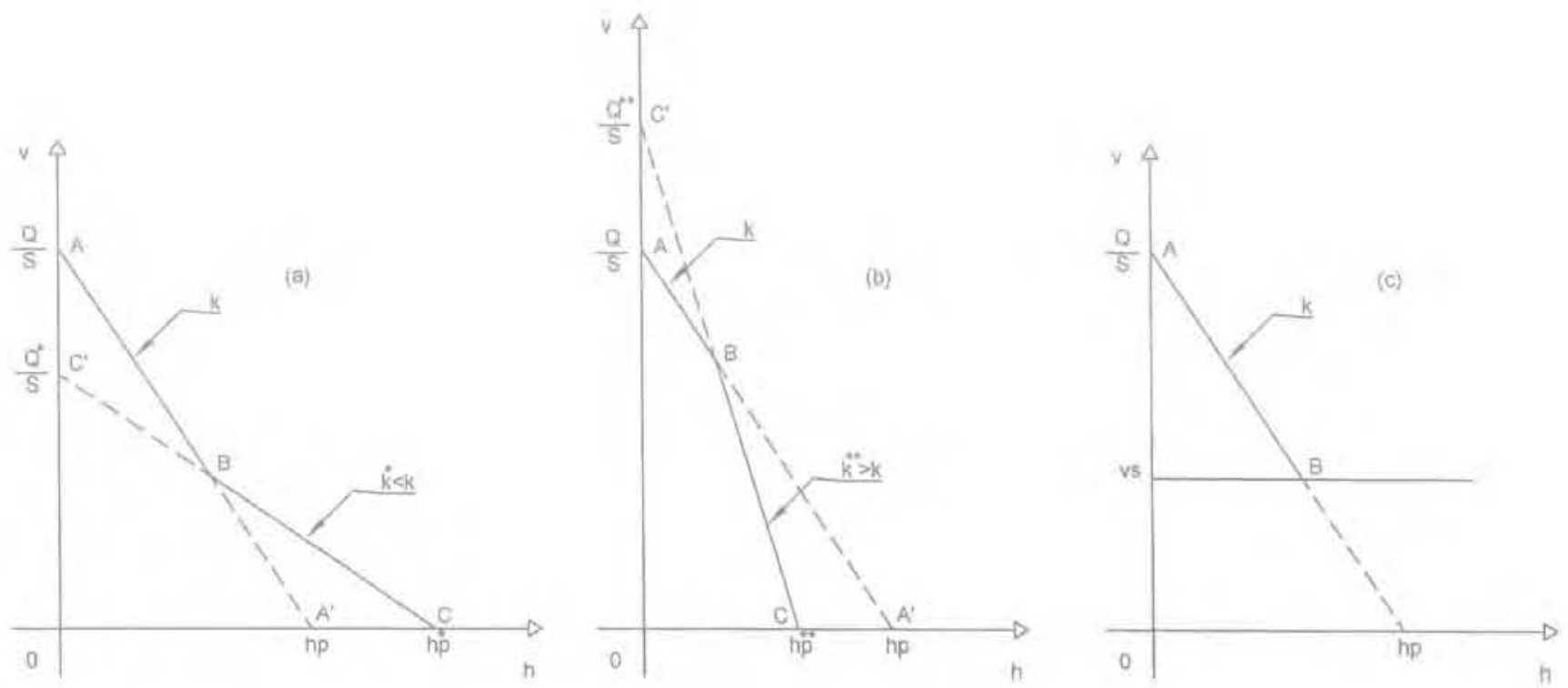

FIG. * Anomalies des courbes de vitesse.
(a) colmatage stabilise
(b) décolmatage stabilisé
(c) colmatage progressif 

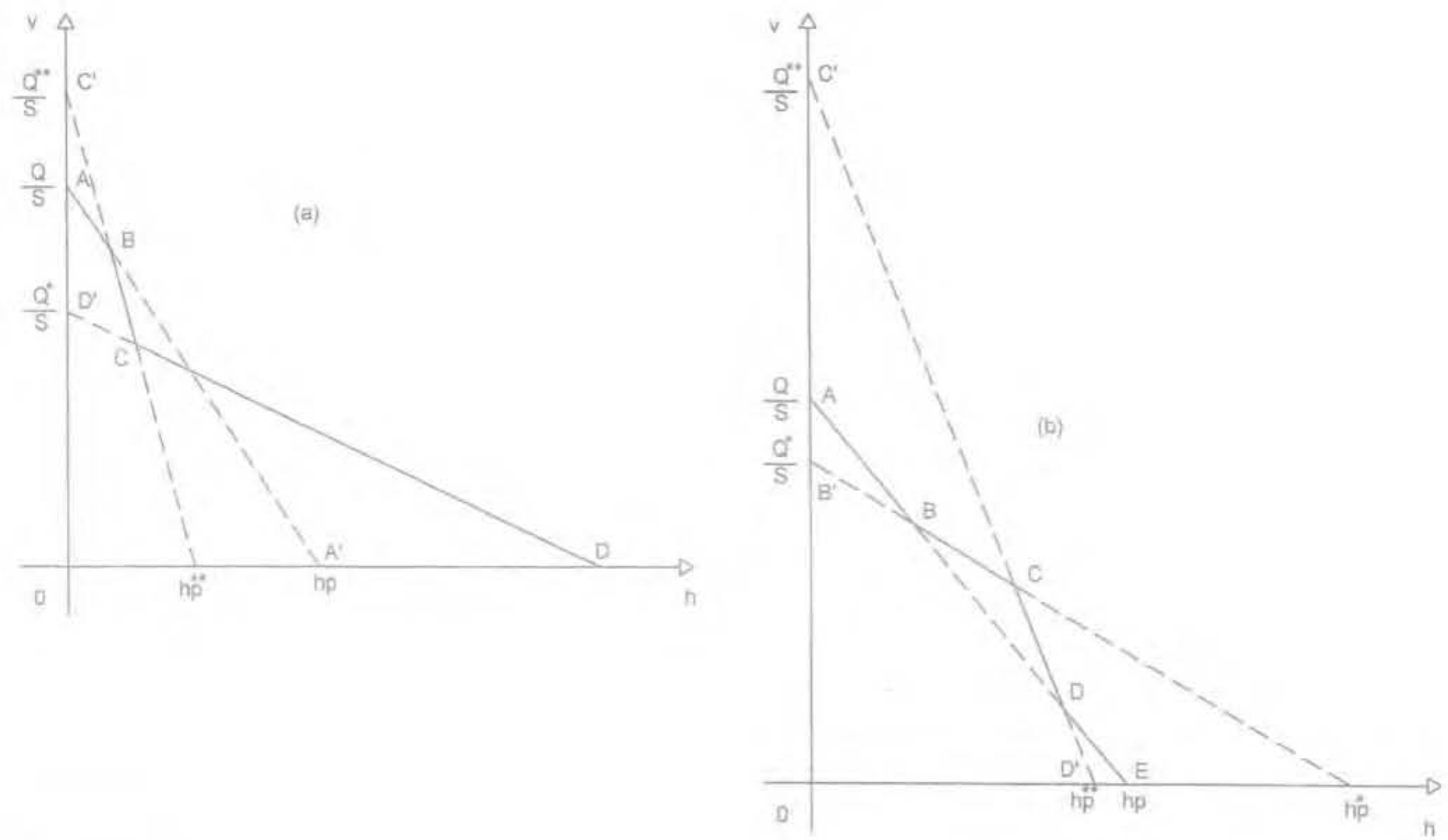

ศ6. A Anomaties des courbes de vitesse.

(a) début d'essai correct suivi d'ur débourrage puis d’un colmatage.

(b) début d'essai correct suivi d'un colmatage puis d'un décolmatage; fiñ d'essai correct.

$$
v_{s}+\frac{m B}{s} k h=v_{0}
$$

soit:

$$
k h=\frac{\left(v_{0}-v_{2}\right) S}{m B}
$$

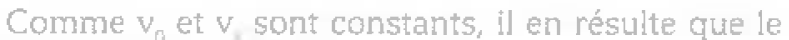
produit kh est également constant.

Le graphe des vitesses comprend ici trois segments et il est facile de voir que :

- le segment $A B$ correspond au terrain non perturbé et permet d'évaluer son coefficient de perméabilité $\mathrm{k}$; - le segment BC traduit un débourrage et conduit à un coefficient de perméabilité $\mathrm{k}^{* *}>\mathrm{k}$ :

- le segment CD représente un colmatage avec un coefficient de perméabilité $\mathrm{k}^{*}<\mathrm{k}$.

Le graphe des vitesses relatives comporte ici quatre segments de droite et correspond tres exactement à la courbe de la figure $7 \mathrm{~d}$. Il est facile er effet de montren que :

- le segment $\mathrm{AB}$ correspond à un début d'essaj correct permettant d'évaluer le coefficient réel $k$;

- le segment BC correspond à un colmatage avec $k^{*}$ $<\mathrm{k}$;

- le segment CD traduit un décolmatage avec $k^{* k}>k$ : - le segment DE prolongement de $\mathrm{AB}$ à un retour à l'état initial non perturbé.

Ces anomalies théoriques ne sont pas des vues de l'esprit, mais sont confirmées par l'expérience comme le montrent les courbes des figures 10 et 11 , extraites d"une étude assez complète réalisée par Fondasol pour EDF sur IIsère à proximité d'Albertvilie entre Cevins et La Batie, et celles des figures 12 et 13 relatives au palais de justice de Lyon. On remarçuera en particulier sur la figure 13b, que linterprétation sous débit nul, après arrêt de la pompe, conduit pratiquement ả la mêrne valeur de $k$ que l"interprétation effectuée à partir de la fígure $13 a$.

Comme on peut le voir, certains essais qui auraient étê, jadis, considérés comme inexplaitables ont conduit à des résultats tout à fait représentatifs grâce à la méthode d'interprétation exposée précédemment.

\section{3}

\section{Cas particulier dit du a débit nul "}

Lorsque l'essai est teminé on arrête la pornpe et l'on doit mesurer en fonction du temps, comme l'indique la norme, l'évolution du niveau de l'eau dans le tube d'écoulement : remontée si on a opéré par prélèvement, ou descente sì on a opéré par injection.

Le phénomène est régi par l'équation (3) dans laquelle on fait $\mathrm{Q}=0$.

On obtient alors:

$$
\frac{d h}{d t}+\frac{m k B}{S} h=0
$$

$$
\text { d'où : } \quad h=h_{0} \exp \left(-\frac{m k B}{S} t\right)
$$


ESSAI LEFRANC REGIME TRANSITOIRE (injection)

E5 $P: 24 \quad B: 3$

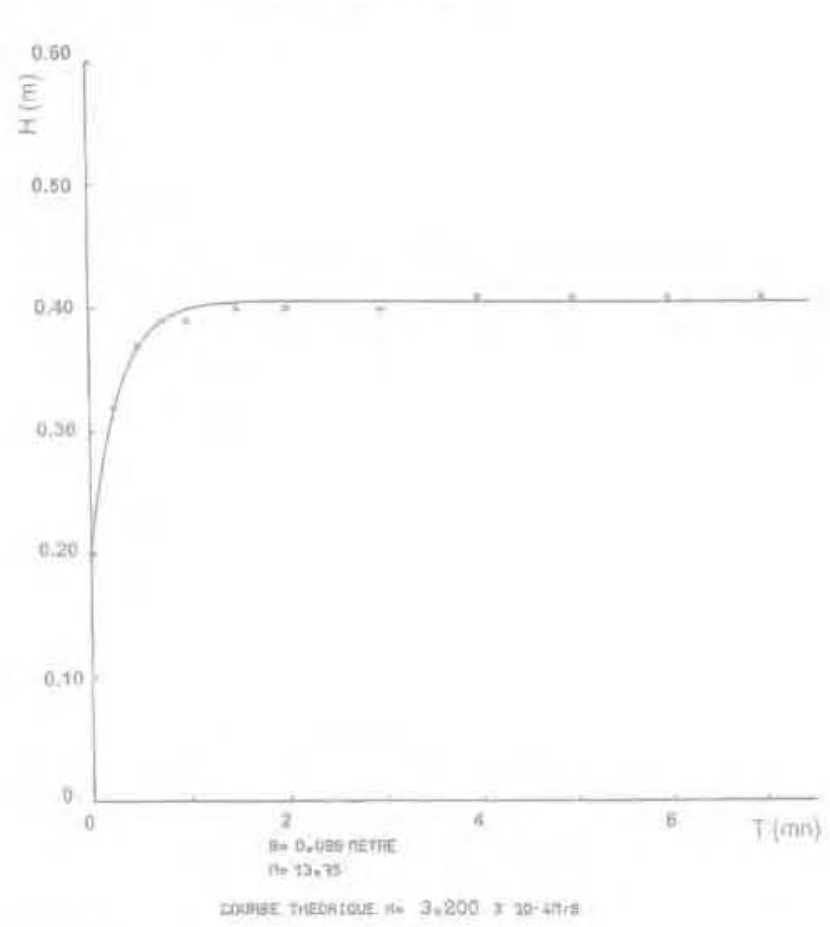

F5. १ดA EDF Cevins.

Ajustement d'une courbe.
ESSAI LEFRANC REGIME TRANSITOIRE (injection)

E5 P:24 $B: 3$

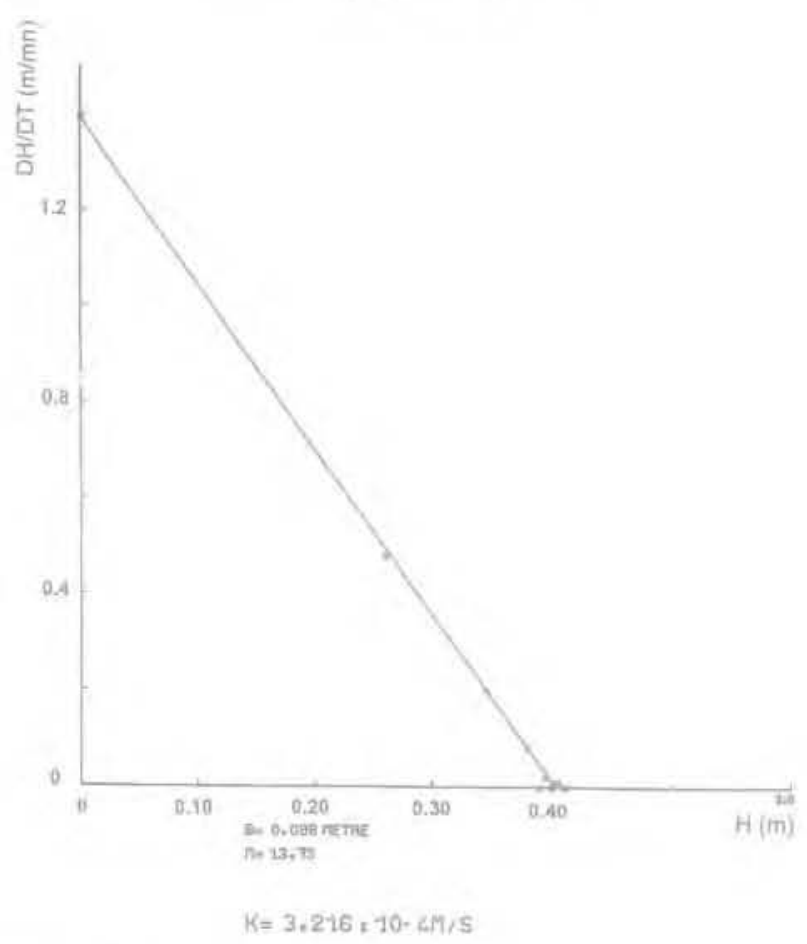

คล. 168 EDF Cevins.

Traitement graphique de l'équation differentielle.
Dans cette relation:

- ho est la charge différentielle au moment de l'arrêt de la pompe.

- t est le temps compté à partîr de l'arrềt de la pompe.

On obtient alors une nouvelle évaluation de $k$ en ajustant la courbe theorique sur les points experimentaux à l'aide du même logiciel qu'en II.1.

On peut également travailer sur le graphe des vitesses relatives $v=\frac{d h}{d t}$ qui est la droite représentée dans le système (v, h), par l'équation (6).

Cet essal sous débit nul est souvent appelé essai sous charge vartable, mais on voit bien qu'i] ne s'agit, en fajt, que d'un cas particulier de l'essai Lefranc puisque, comme on vient de le voir, on l'interprète en faisant $Q=0$ dans les formules générales.

Cet essail est spécifiquement utilisé dans le cas de terrains peu perméables lorgque le débit que peut absorber le sol est trop faible.

La méthode par injection consiste alors à remplir le forage et mesurer l'abaissement du niveau de l'eau en fonction du temps, et la méthode par prélèvement consiste à vider partiellement le forage et à relever la remontée de l'eau.

Cette dernière méthode a fait l'objet dans les années soixante de la mise au point par un ingénieur de Solétanche, J. Brillant, d'uñ dispositif très ingénieux et très pratique, et l'essai correspondant a été appelé essai Brillant (J. Brillant 1960 et 1966).
Malheumeusement cef essai est tombé dans l'oubli et il n'est pas certain que l'appareillage existe encore sauf peut-être dans les caves de Solétanche!

Il existe, certes, des essais en forage, appelés essais à charge variable qui ressemblent à des essais Lefranc. mais qui ne sont pas des essais Lefranc, et qui relèvent de normes différentes car ils ne sont applicables qu'aux sols de très faibles perméabilités.

Il s'agit:

- de l'essai à charge variable en Forage ouvert applicable à la mesure de coefficients de perméabilié compris entre $10^{-5}$ et $10^{-5} \mathrm{~m} / \mathrm{s}$ et régi par la norme NEX.30.423 (septembre 2002) :

- de l'essai à charge variable en forage fermé applï cable à la mesure de coefficients de perméabilités infién rieurs à $10^{-13} \mathrm{~m} / \mathrm{sec}$ et régi par la norme NFX.425 (aoủ 2002).

Ces deux essais nécessitent des dispositifs experimentaux et des corrections de mesures spécificues, en particulier la correction de la viscosité dynamique de l'eau en fonction de la température. Si l'interprétation du premier est, peut être un peu abusivement, la même que celle de l'essai Lefranc sous débit nul, l'interprétation du second que l'on appelle quelquefols choc hydraulique est trés differente et beaucoup plus complexe, et fait intervenir la compressibilité du sol et celle de l'eau.

Ne s'agissant pas d'essais Lefranc, je me limiterai à cette simple évocation, car tout développement nous 
ESSAI LEFRANC REGIME TRANSITOIRE (injection)

$E 2 P: 44.58: 0$

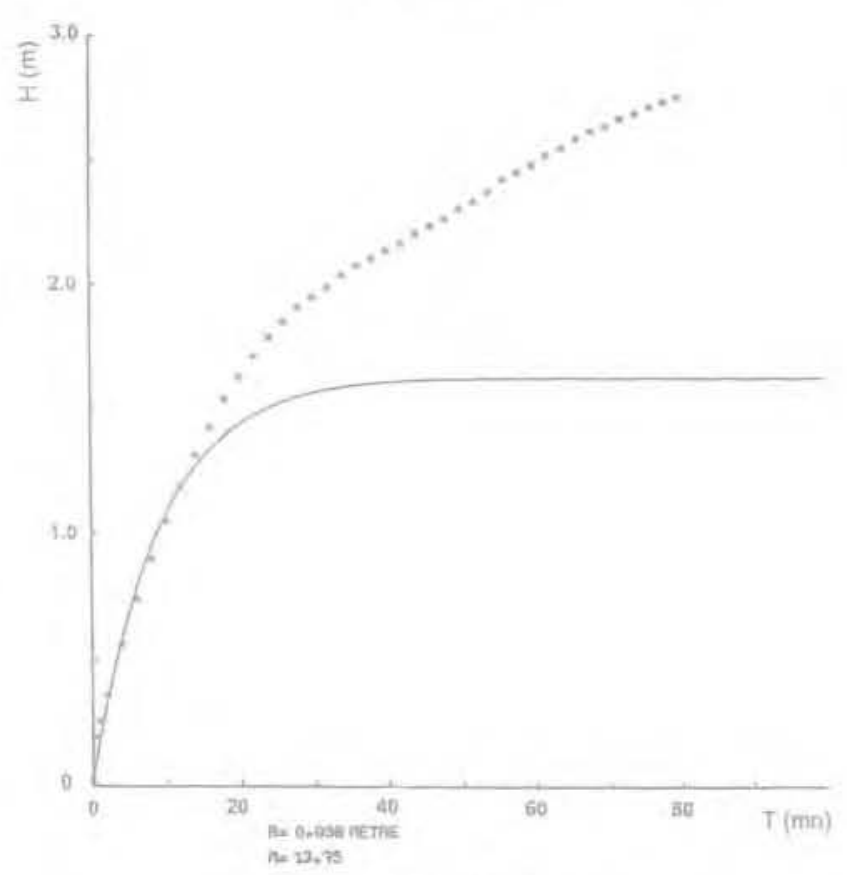

tourae nenatue his 0,100130 - Lhis

FG. 19A EDF Cevins.

Ajustement d’une courbe théorique.

ESSAI LEFRANC REGIME TRANSITOIRE (pompage)

SC5 P a3. 70 MEBIT: 5 51 10-4M3/S

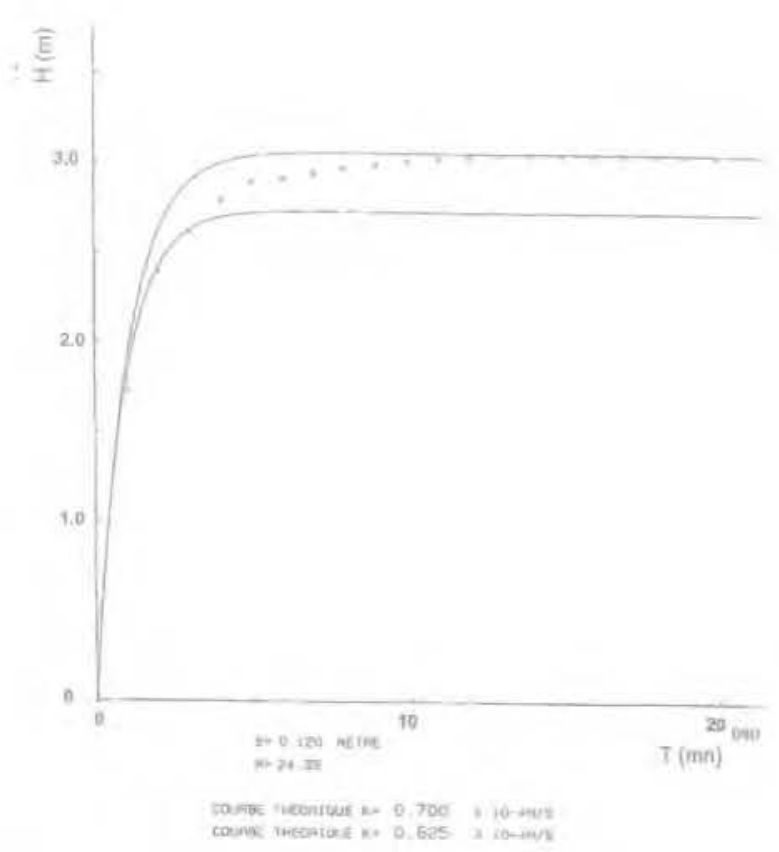

Fa 12A Palais de Justice de Lyon.

Ajustement d'une courbe théricue (lẻger colmatage\}.
ESSAI LEFRANC REGIME TRANSITOIRE (injection)
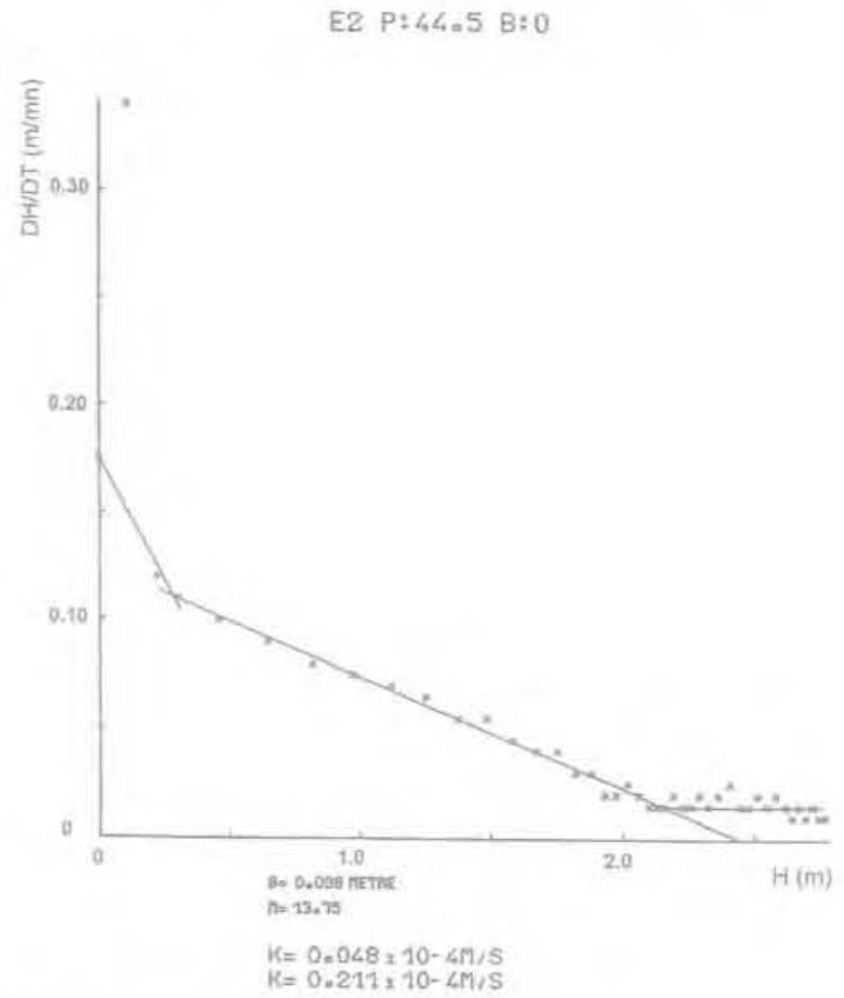

Fis. 118 EDF Cevins.

Tratement graphique de l"éguation différentielle.

ESSAI LEFRANC REGIME TRANSITOIRE (pompage) SCS P 23 TOM AEMOMTEE

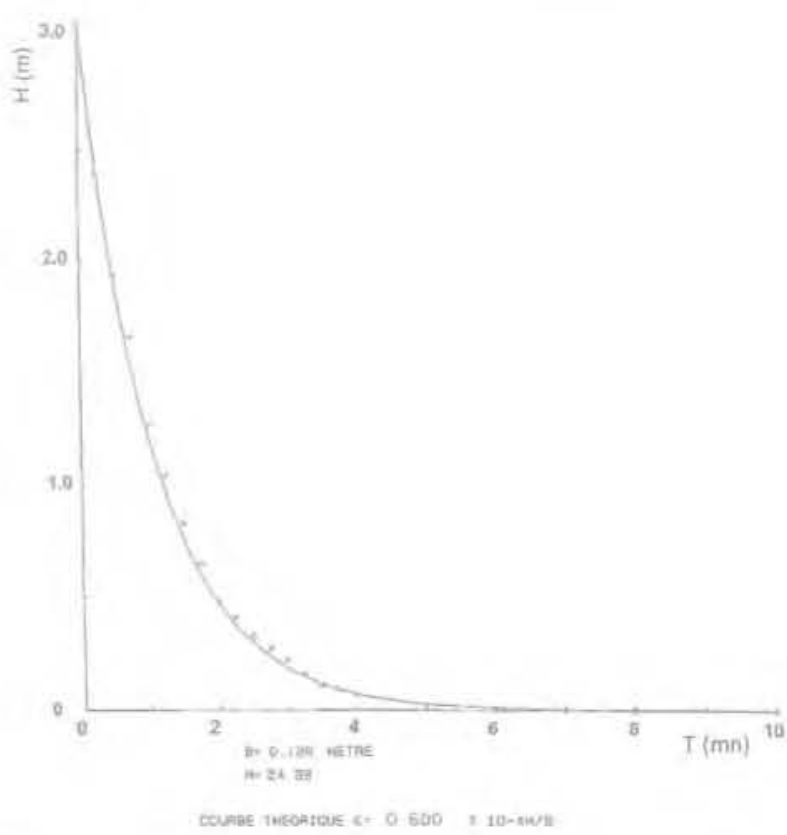

La. 198 Palais de Justice de Lyon - débit nul. Apres atrêt des pompes. 
ESSAI LEFRANC REGIME TRANSITOIRE (pompage)

SCG P: 24 . 46M DEBIT: 2 . B5 10-4M3/S

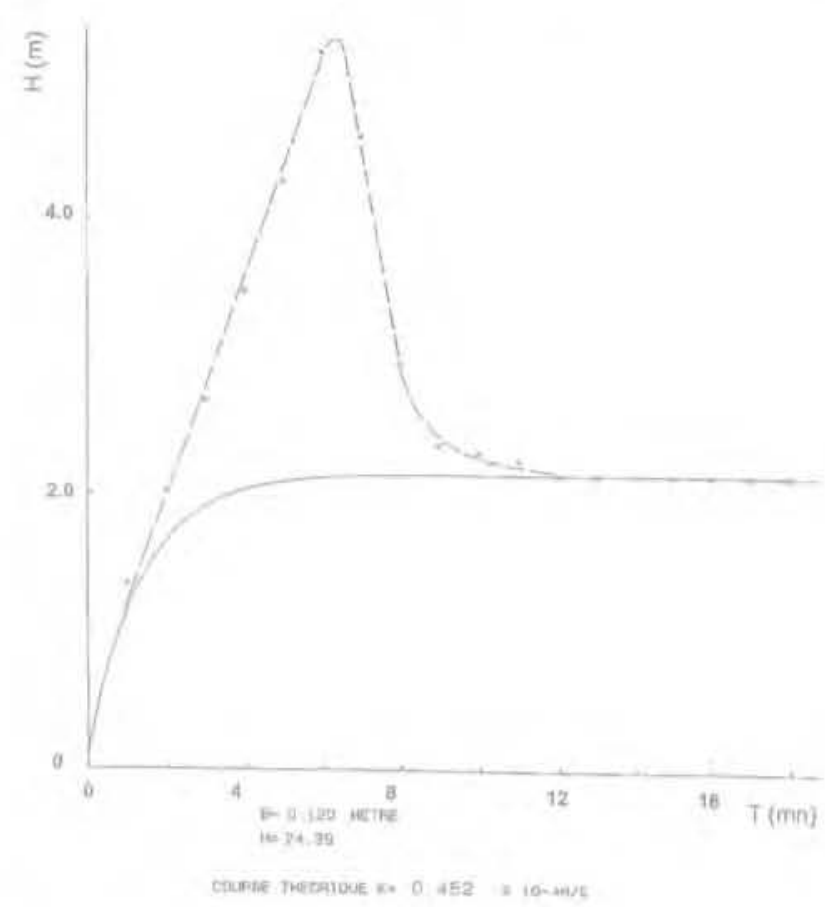

ME. 13. Palais de Justice de Lyon.

Colmatage suivi d'un décolmatage et d'une stabilisation.
ESSAI LEFRANC REGIME TRANSITOIRE (pompage)

SC9 P. 24 . 4 MM REMONTEE

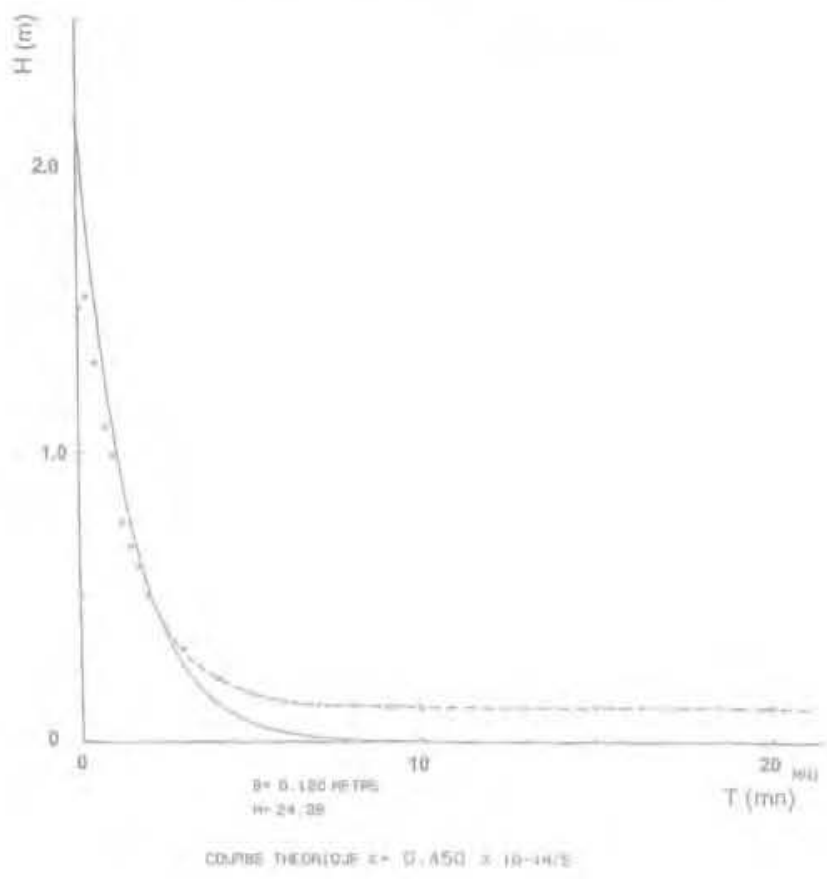

Flō 138 Palais de Justice de Lyon - débit nul. Après arrêt dles pompes. entrainerait trop loin, mais je rappelleraj que dans la prenière phase de l'essai Lefranc la charge est variable et qu'elle n'est constante que dans la deuxiène phase, lorsque le régime permanent est atteint.

\section{3}

\section{Analyse de cas réels}

Nous allons analyser ici les résultats obtenus sur deux grands chantiers.

Le premier conceme une étude hydrogéologicue qui a permis de comparer des essais Lefranc classigues réalisés par injection et des essais Lefranc réalisés par pompage dans une cavité de grande dimension.

Le second conceme l'étude du rabattement permanent d"une rappe qui a permis, d'une part, de comparer les résultats d'essais Lefranc classiques avec ceux d'un pompage traditionnel et d'autre part, de comparer les débits de rabattement prévus dans l'étude et les débits réellement obtenus.

\section{1}

\section{Aménagement Romanche-Isère (EDF)}

Cette opération située entre Vizille et Sêchilienne a comporté la réalisation de :

- trois forages de reconnajssance géologique carottés ả la colonne de $120 / 140 \mathrm{~mm}$ et descendus entre 36 et
40 mètres de profondeur, puis éguipés de piézomètres de $40 / 49$ rnm

- trois forages destructifs de $120 / 140 \mathrm{~mm}$ pour la réalisation d'essais Lefranc;

- un puits de pompage de $500 \mathrm{~mm}$ de diamètre descendu à 30 mètres de profondeur et assorti de 16 piézomètres satellites, dont 10 furent arrêtếs à 15 mètres et 6 descendus à 26 mètres. Ce puits étaít destiné à réaliser des essais de pompage sous fort débit.

Les terrains traversés sont les alluvions de la Fomanche constitués par une succession de sables pius ou moins graveleux et de graves sableuses, âlé ments centimétriques à décimétriques, pouvant être localement limoneuses avec des passages de gros blocs.

I] S'agit donc d'un matériau granulaire à granulométrie assez variable et présentant au-dessous de 16 mètres. une tendance légèrement plus argilo-limoneuse (Fig. 14).

La principale particularité de l'opération a résidé dans la réalisation du puits et des pompages qui a comporté trois phases.

Premiere phase :

- forage du puits à l'hammergrab avec tubage de 500 mm jusqu'à 15 mètres de profondeur:

- éguipement du puits avec un tube crépiné de $350 \mathrm{~mm}$ de diamètre, filtre en giavillon et développement à l'émulseur: 
- remontée du tube extérieur de 500 mm sur une hauteur de 5 mètres :

- pompage sous un débit de $480 \mathrm{~m}^{3} /$ heure pendant 20 heures avec mesures dans le puits et les piézomètres.

\section{Deuxième phase}

- extraction du tube crépiné de $350 \mathrm{~mm}$;

- reprise du forage à l'hammergrab avec tubage de $500 \mathrm{~mm}$ jusqu'à sa profondeur définitive de 30 mètres;

- équipement du puits avec le tube crépiné de $350 \mathrm{~mm}$ et développement à l'émulseur"

- remontée du tube extêrieur sur une hauteur de 10 mètres ;

- pompage sous un débit de $460 \mathrm{~m}^{3} / \mathrm{heure} \mathrm{pendant}$ 62 heures avec mesures dans le puits et les piézomètres.

\section{Troisième phase:}

- extraction complète du tubage de $500 \mathrm{~mm}$.

- équipement définitif du puits :

- pompage classique dans le puits crépiné sur toute sa hauteur.

Ce qui est intéressant dans celte procédure, c'est que, dans les deux premières phases, le puits n'était pas crépiné sur toute sa hauteur mais seulement à sa base, sur une hauteur de 5 mètres pour le premier pompage et de 10 metres pour le second, et que cette partie crépinée était hydrauliquement isolée par le tube de forage $\Phi 500$ dont le sabot n'était pas débordant.

Dans les trois phases, le pompage a été interprété de manière classicue à partir des piézomètres satellites ce qui a permis d'évaluer la transmissivité de l'aquifère mais pas son coefficient de perméabilitá puisque l'épaisseur de cet aquifère était très importante et non précisée (plus de 50 mètres).

En revanche, dans les deux premlères phases, compte tenu des dispositions adoptëes et décrites cidessus, le phénomène hydraulique dans le puits en cours de pompage est identique à celuj d"un essaj Lefranc.

On a donc étudié l'évolution du niveau de l'eau dans le puits comme s'il s'agissait d'essais Lefranc avec des cavités de grandes dinensions (diamètre 0,50 mètre et longueurs de 5 et 10 mètres' et sous des débits trés élevés, ce qui n'est évidemment pas courant.

Tous les essais Lefranc, qu'il s'agisse des essajs classiques dans les forages spécificues en $120 / 140 \mathrm{~mm}$ ou des deux essais dans le puits de pompage, ont été anaIysés selon les méthodes exposées précédemment.

Dans le premier pompage, la courbe d'évolution de la charge en fonction du temps présente, dès le départ, des anomalies qui évoquent un phénomène de colmatage. Il n'a pas été possible de trouver une courbe théorique qui s"ajuste sur les points expërimentaux mais les six premiers points correspondant aux dix premières minutes, semblent, à la rigueur, pouvoir àtre considérés comme voisins d'une courbe théorique. Une évaluation du coefficient de perméabilité à partir de ces points a donné :

$$
\mathrm{k}=3.10^{-2} \mathrm{~m} / \mathrm{s}
$$

Aubout de 10 heures de pompage on voit apparaître un palier d'une durée de 4 heures (Fig. 15).
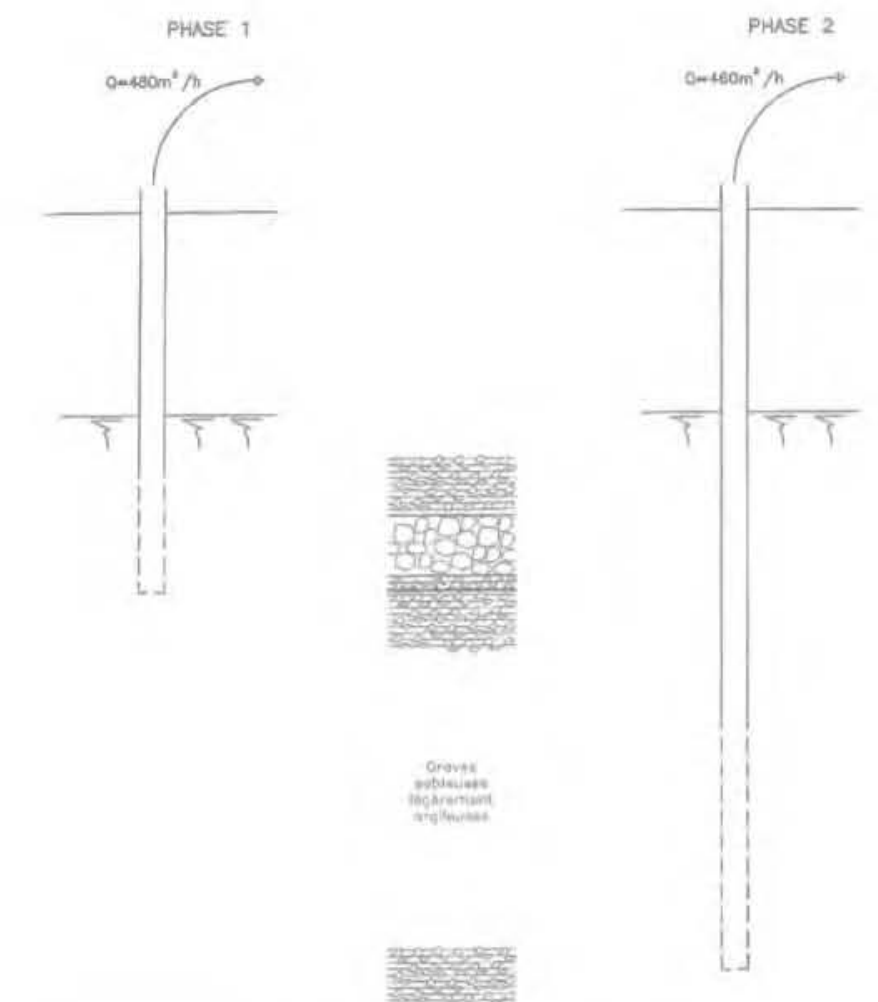

FIG. 14

EDF aménagement Romanche - Isère. Vizille - Sechilienne.

Par curiosité on a évalué le coefficient de perméabilité à partir de ce palier comme s'il s'agissait du régime permanent dans un essai non perturbé. On a ainsi obtenu:

$$
\mathrm{k}=2.10^{-2} \mathrm{rn} / \mathrm{s}
$$

L'écart n'est donc pas très significatif, mais il faut cire que la cavité de filtration constituée par la base du puits était particulièrement mal placée puisque sa mojtié supérieure se trouvait dans des sables, graviers et galets légèrement argileux avec des blocs et que sa moitiê inférieure étajt située dars ur amas de blocs (Fig. 14-phase 1). I] n'est donc pas étonnant cu'on ait obtenu ur coefficient de perméabilité élevé at il n'est pas impossible que les anomalies constatées aient pu provenir de l'établissement d'un régime turbulent.

On peut donc globalenent retenir a ce niveau

$$
2.10^{2} \leq k \leq 3.10^{2} \mathrm{~m} / \mathrm{s}
$$

Mais le parallélisme des deux segments inclinés situés de part et d'autre du palier n'est pas sans rappeler le phénomène d’égouttement, ce cul complique quelque peu les choses et conduirait à une précision illusoire si l'on voulait faire intervenir ce phénomène dans le dépouillernent.

Dans le dewxième pornpage, réalisé avec une cavité de 10 mètres de hauteur et dont le centre est ả 25 mètres de profondeur, i] n'a pas étê, Jà non plus, possible d'ajuster une courbe théorique sur les points expérimentaux bien que l'on se trouve dans une zone plus homogène.

A partir de la $53^{*}$ heure de pompage, on a obtenu un palier mais celui-ci correspond ầ un matériau perturbé par un phẻnomène de colmatage. L“interprétation a pu se faire en adoptant le schéma de la figure $7 b$, 


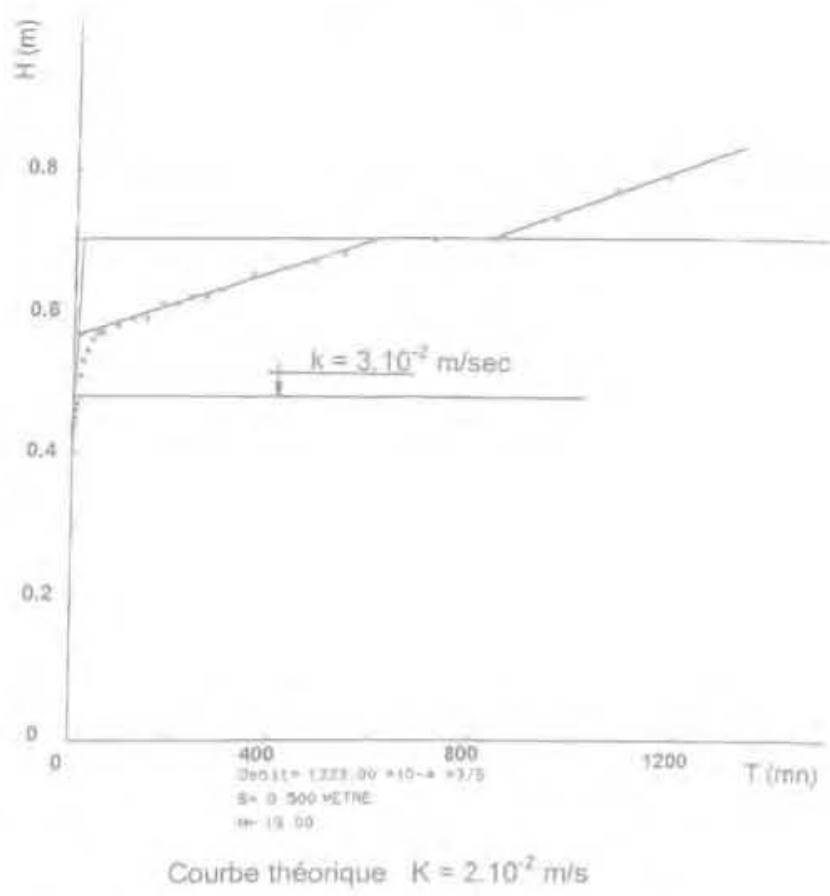

FI6. 13 EDF Séchilienne-cavité 10 à 15 mètres.

la courbe théorique étant déterminée à partir des huit premiers points (Fig. 16). Le coefficient de perméabilité correspondant est alors:

$$
\mathrm{k}=7,5.10^{-3} \mathrm{~m} / \mathrm{s}
$$

Mais le colmatage a dû être très important car après l'arrêt des pompes, il n'a pas été non plus possible d'ajuster la courbe théorique sur l'ersemble des points expérimentaux (Fig. 17). L'ajustement n'a pu se faire que sur les premiers points de la descente et a donné :

$$
\mathrm{k}=7.10^{-7} \mathrm{~m} / \mathrm{s}
$$

ce qui confirme le colmatage.

En ce qui concerne les essais Lefranc classiques, les courbes expérimentales sont un peu moins perturbées, et les colmatages que l'on a observés sont du type de cetux de la figure $7 a$.

Le nombre total d'essais Lefranc classiques réalisés par injection est de 82, mais certains de ces essais ont fait l'objet de plusieurs paliers de débit.

Il en résulte que le nombre de niveaux testês était de 27. c'est-k̀-dire que nous avons donné 27 valeurs de coefficient de perméabilité.

Parmi ces 27 valeurs:

-2 ćest-à-dire $7.4 \%$ se situaient dans la tranche de $10^{-6} \mathrm{~m} / \mathrm{s}$;

-2 se trouvajent dans la tranche des $10^{-4} \mathrm{~m} / \mathrm{s}$;

- 17, c'est-à-dire $63 \%$ appartenaient à la tranche de $10^{-3} \mathrm{~m} / \mathrm{s}$;

- 6, c'est-à-dire $22,2 \%$ étaient dans la tranche des $10^{-2} \mathrm{~m} / \mathrm{s}$

Les deux valeurs de l'intervalle $10^{-6}$ peuvent être négligées car les courbes obtenues, linéaires en fonc-

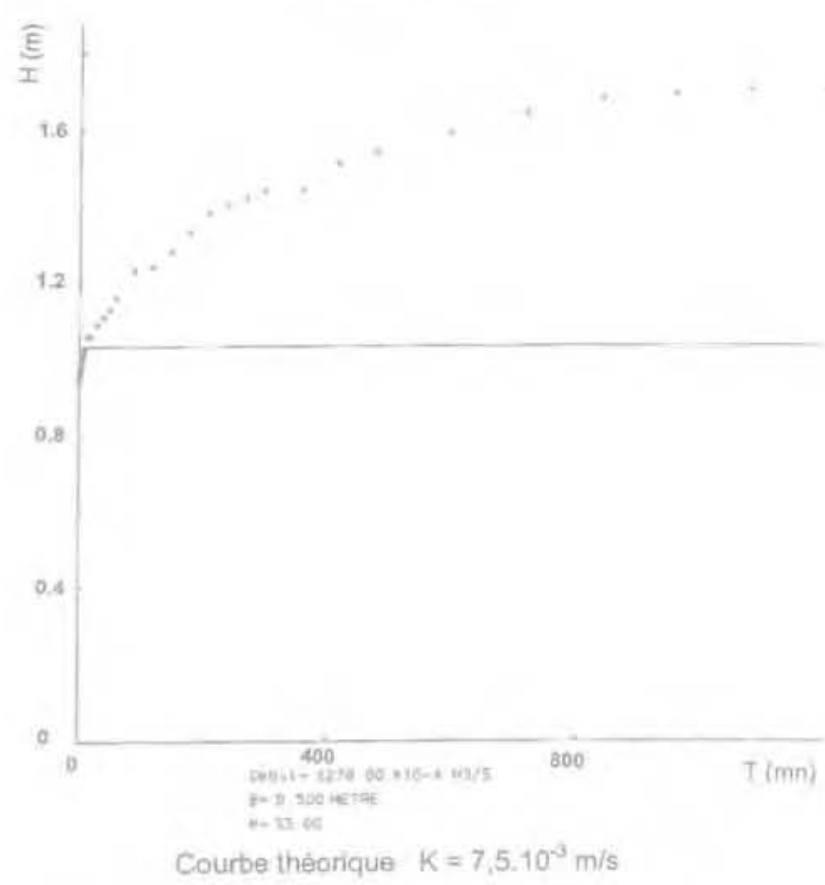

Fit. 16 EDF Séchilienne - cavité 20 à 30 mètres.

tion du temps, trađuisent un colmatage immédiat, et cela sans aucune ambiguilté.

Sur l'histogramme logarithmique de la figure 18, nous avons reporté la position des deux pompages et nous constatons que, globalement il n'y a pas de contradiction flagrante entre les cleux expérimentations. Mais il convient néanmoins de tempérer un peu l'optimisme fallacieux que peut donner cet histogramme logarithmique. C'est pourquoi nous donnons sur la figure 19, les histogrammes arithmetiques pour les deux épaisseurs testées, 0 à 15 mètres comespondant au porpage 1, et 15 à 30 mètres correspondant au pompage 2.

Nous constatons un étalement très important des valeurs de $\mathrm{k}$ qui n’est toutefois pas anomal pour ce parametre.

On remarque alors que, pour la tranche 10/15 mètres, la fourchette de perméabilité donnée par Le pompage I n'encadre qu'un seul essal Lefranc ef que globalement l'essai par pormpage donne des valeurs supérieures aux essais classiques dans un rapport de 1 à environ, c'est-à-dire presque une puissance de 10. Mais ce résultat n"est pas strprenant, car comme nous l'avons vu précédemment, la cavité de 5 mètres de hauteur est située pour moitié dans une zone de blocs très perméables dans laquelle le régime d'écoulement n'est peut-être pas laminaire.

En revanche, au-dessous de 15 mètres, les résultats du pompage 2 sont assez voisins de ceux des essais Lefranc. Nous avons en effet dans l'ensemble:

- essai Lefranc : $1.10^{-3}<\mathrm{k}<5.10^{-3} \mathrm{~m} / \mathrm{s}$ :

- pompage; $k=4$ à $7.10^{-3} \mathrm{~m} / \mathrm{s}$ 


\section{ESSAI LEFRANC REGIME TRANSITOIRE}

POMPAGE $2 \quad Z: 20 m$ remontée

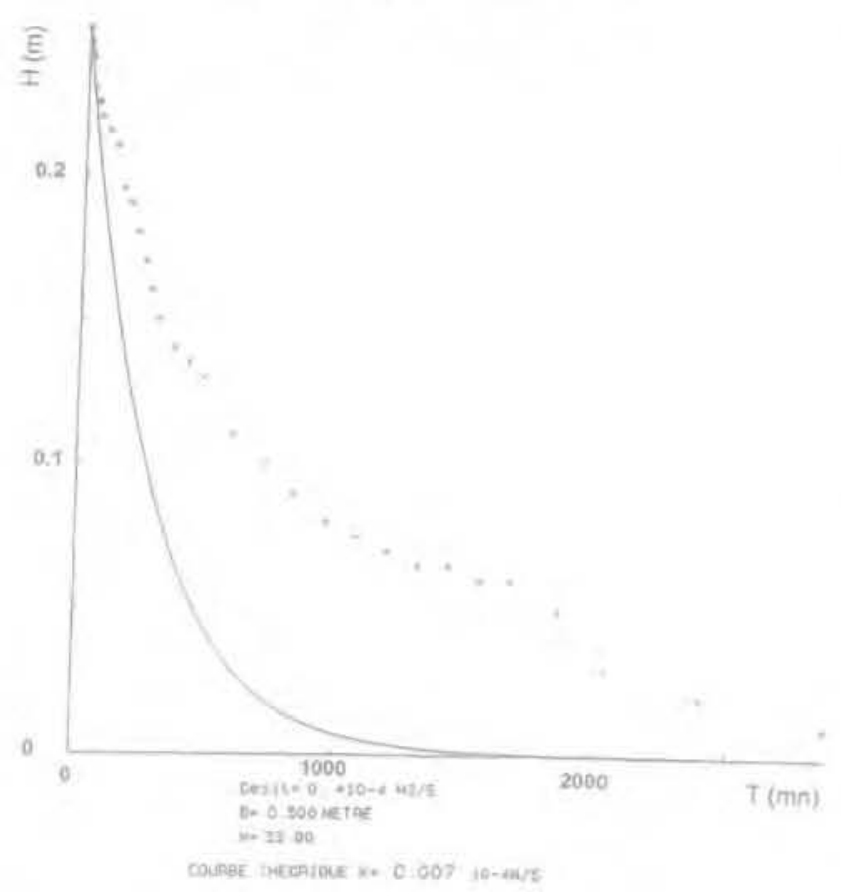

*19. 17 EDF Séchilienne-remontée.

La concordance est encore meilleure si on considère l'ensemble du site, toutes profondeurs confondues, ce qui n'est pas étonnant puisque la cavité du pompage 2, malgrê sa hauteur de 10 mètres est entièrement siluée dans une formation de grave sableuse localemert, mais faiblement limoneuse, et que c'est dans cette formation que l'ensemble des essais Lefranc a étẻ réalisé.

\section{2}

\section{Palais de justice de Lyon}

L'actuel palais de justice de Lyon, en service depuls quelques années, comporte un sous-sol de parking de 21 mètres de profondeur environ, dont 18,80 métres sous la nappe.

La mise hors d'eau de ce parking par radier général et cuvelage étanche a été rejetée par les concepteurs compte tenu de la sous-pression quj se serait exercée sous le radier.

La solution qui a été retenue fut celle d'un radier drainant avec pompage permanent sous réserve que le débit de pompage nécessaire pour rabattre la nappe de 18,80 mètres n'entraine pas des frais de maintenance susceptibles de remettre le projet en cause. Il importait donc pour cela de déterminer le débit d'épuisement avec la plus grande fiabilitế possible.

Nous avons donc réalisé un programme qui a comporté :

- des forages de reconnaissance classique, carottés et destructifs :

- des essais Lefranc:

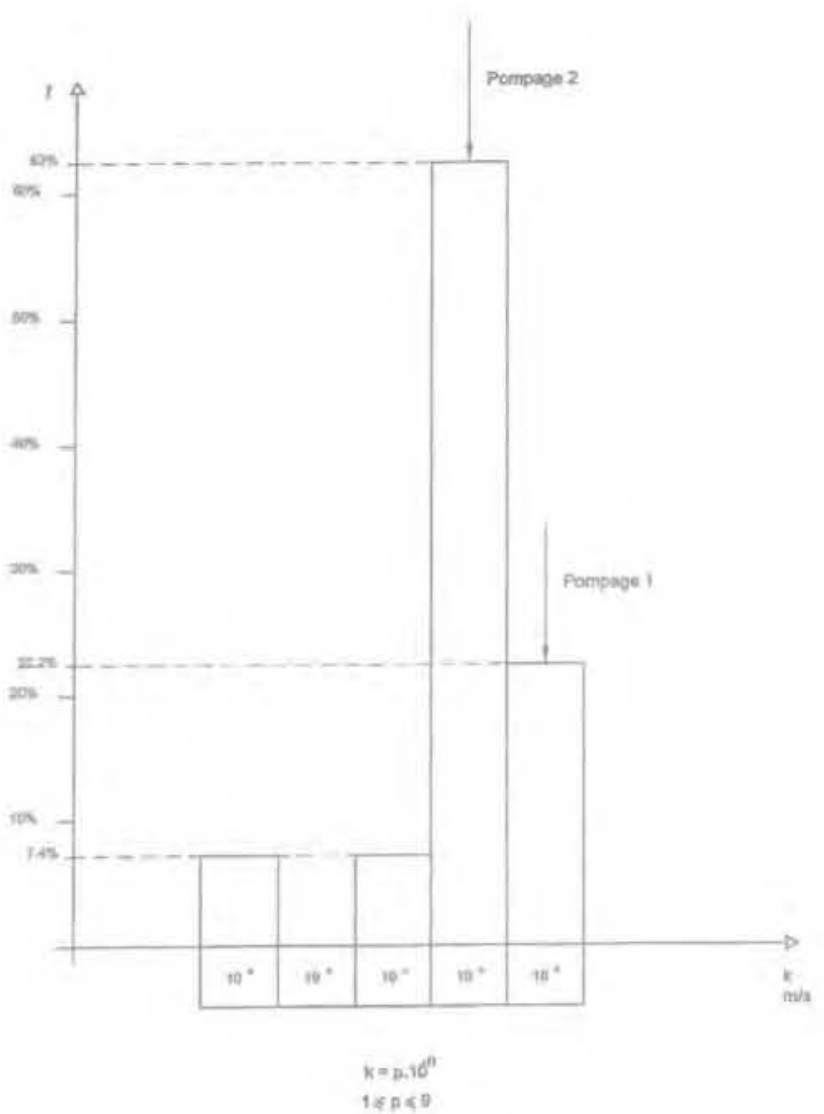

FIG. 18 Répartition globale des coefficients de perméabilité. EDF Romanche - lsère (Séchilienne).

- deux essais de pompage, l'un, préliminaire de très courte durée, l'autre de 10 heures.

La recomaissance a fait apparaitre, sous les alluvions grossières du Rhône, la formation molassique bien connue dans la région lyonnaise et c'est dans cette dernière formation qu'ont été réalisés les essais de perméabilité, car nous avons estimé que les alluvions grossières du Rhône pouvatent être considérées comme infiniment perméables par rapport à la malasse. De plus, le radier drainant devait être descendu dans la partie supérieure de cette molasse dans laquelle devait être fichée l'enceinte en paroí moulée destinée d'une part à assurer la stabilité des parois de la fouille pendant les travaux et d'autre part à constituer les murs extérieurs du parking en sous-sol.

Le nombre d'essais Lefranc a été très faible eu égard à l'importance du problème, onze seulement. mais il faut dire qu"iłs nont été réalisés que dans la molasse.

Ces essais ont donné des coefficients de perméabilité tels que :

$$
2.5 .10^{-5} \leq \mathrm{k} \leq 10.10^{-5} \mathrm{~m} / \mathrm{s}
$$

Mais sept de ces essais ont été réalisés par pompage et quatre par injection. Nous avons obtenu:

- essais par injection

$$
5.10^{-5} \leq \mathrm{k} \leq 10.10^{-5} \mathrm{r} / \mathrm{s}
$$

- essais par pompage :

$$
2,5.10^{-5} \leq \mathrm{k} \leq 9.10^{-5} \mathrm{~m} / \mathrm{s}
$$




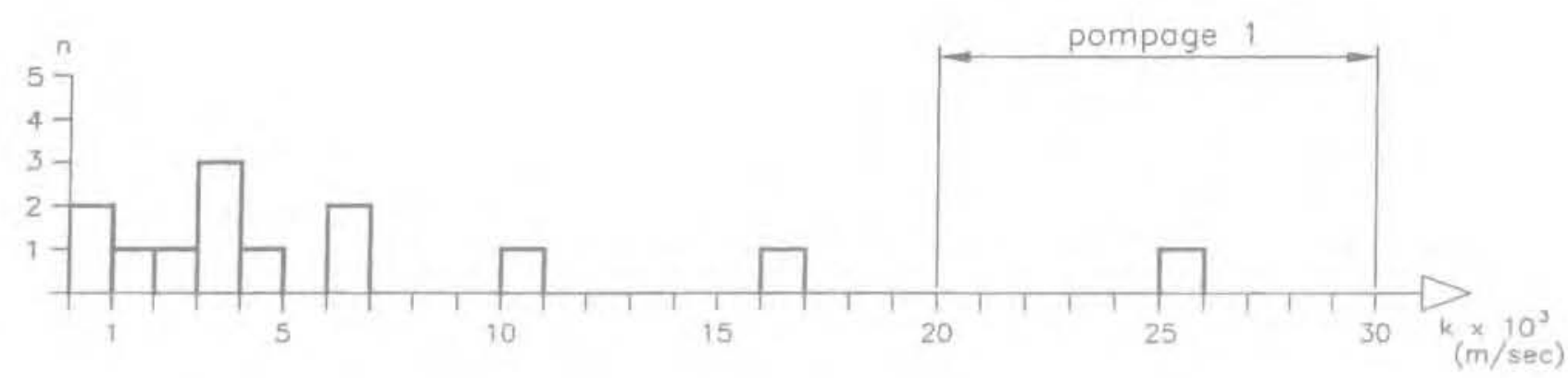

a. Profondeur inferieure ò 15 mètres

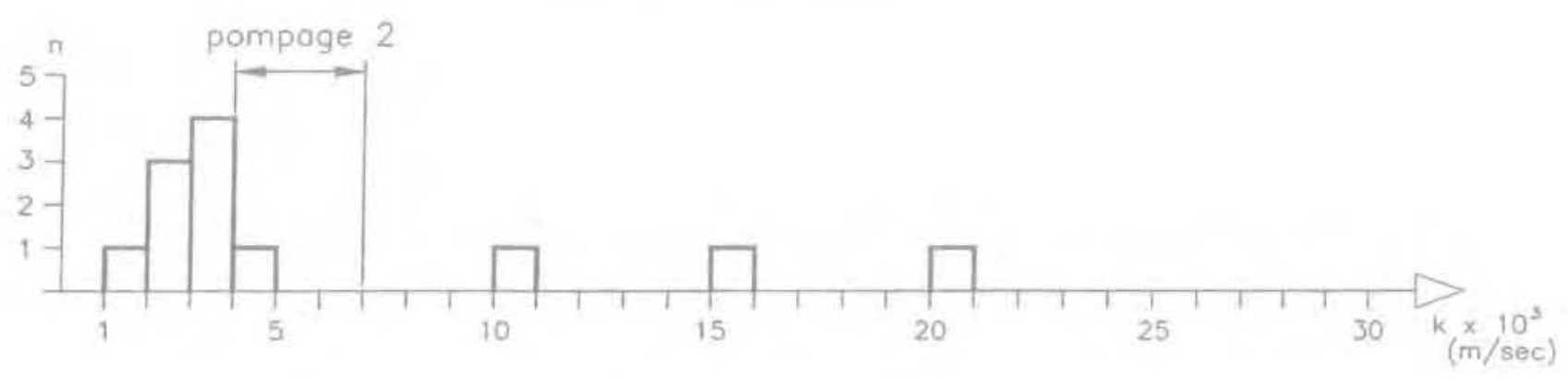

b. Profondeur supérieure à 15 mètres

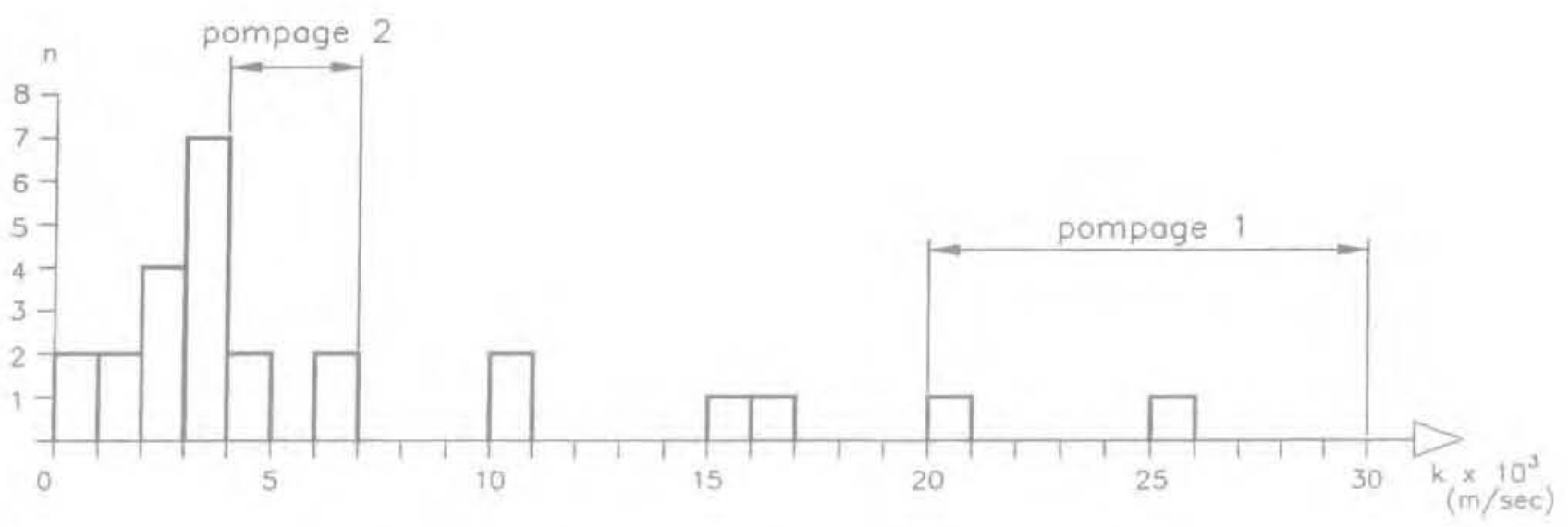

c. Toutes profondeurs confondues

FG. 19 Répartition détaillêe des coefficients de perméabilité. EDF Romanche - Isère [Séchilienne).

On voit qu'il n'y a pas de différence significative entre les deux méthodes.

Les essais de pompage réalisés dans un puits assorti de trois piézométres on donné respectivement:

$$
\mathrm{k}=4,8 \cdot 10^{-5} \text { et } 5 \cdot 1 \cdot 10^{-5} \mathrm{~m} / \mathrm{sec}
$$

On voit donc que les essais Lefranc et les essais de pompage ont donné des résultats du mêne ordre ce grandeur avec toutefois des valeurs légèrement plus élevées pour les essais Lefranc.

Cette excellente corTespondance provient de ce que, d'une part le sol étudié étaít constitué d'éléments fỉns et que d'autre part le diamètre des forages pour essais Lefranc était assez voisin de celui du puits $1 \Phi 120 / 140\}$.
Deux analyses granulométriques réalisées dens l'un des forages pour essais Lefranc ont permis d"appliquer la formule de Hazen qui a donné :

$$
k=1.2 \cdot 10^{-5} \text { à } 4,2 \cdot 10^{-5} \mathrm{~m} / \mathrm{s}
$$

Nous avons aiors adopté, comme résultat des mesures, la valeur $\mathrm{k}=5.10^{-5} \mathrm{~m} / \mathrm{s}$ et nous avons admis que cette valeur correspondait au coefficient de perméabilité horizontale $\mathrm{x}_{\mathrm{n}}$. Sans parler véritablement d'étude bibliographique, une enquête nous a permis de considérer cu'un coefficient d'anisotropie de 5 étajt une valeur vraisemblable. On a donc retenu finalement :

$$
\begin{aligned}
& k_{h_{h}}=5 \cdot 10^{-5} \mathrm{~m} / \mathrm{s} \\
& k_{\mathrm{s}}=1.10^{-5} \mathrm{~m} / \mathrm{s}
\end{aligned}
$$




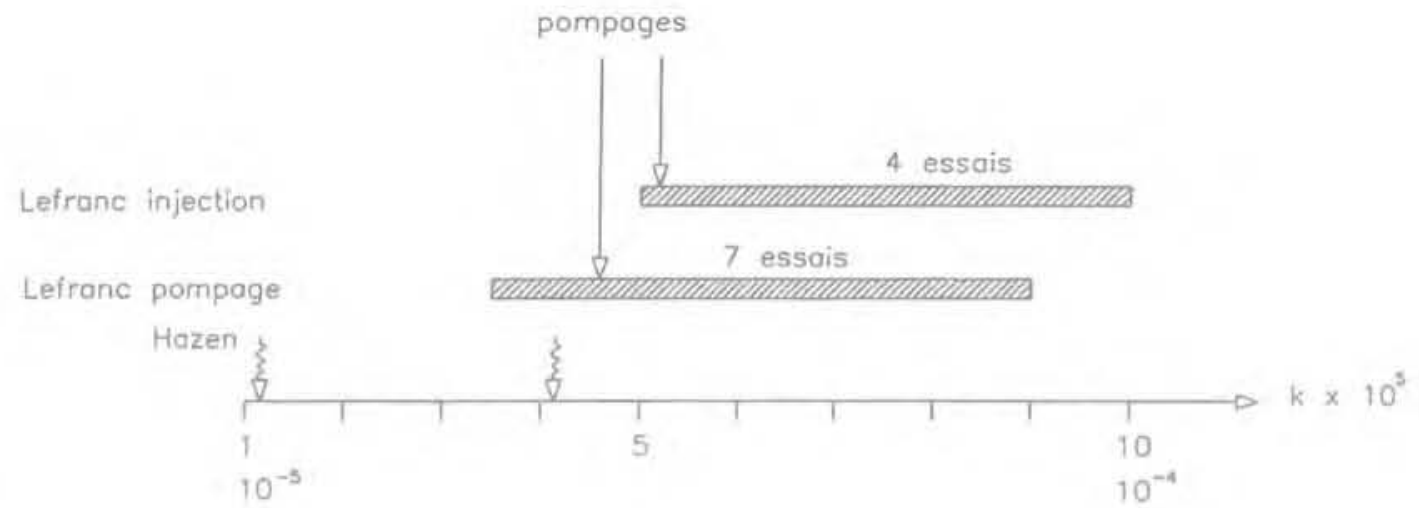

HG qo Comparaison entre essaj de pompage et essai Lefranc. (Palajs de Justice de lyon).

En appliquant aux coordonnées spatiales l'affinité classique bien connue qui permet de transformer l'équation de continuité en Julieu anisotrope en équation de Laplace, nous avons obtenu entre le débit 0 dans le milieu réel et le débit $Q^{\prime}$ dans le milieu transformé, la relation:

$$
Q=Q \sqrt{\frac{h_{h}^{2} k_{v}}{k^{3}}} \quad \text { aveck arbitraire }
$$

Nous avons alors imposé la condition $\mathrm{Q}^{\prime}=\mathrm{Q}$, ce qui a entraîné :

$$
\mathrm{k}=\sqrt[3]{\mathrm{k}_{\mathrm{h}}^{2} \mathrm{k}_{\mathrm{y}}}=3.10^{-5} \mathrm{~m} / \mathrm{s}
$$

Enfin, une enquête relative à deux chantiers voisins analogues nous a permis d'acquêtir des données à partir desquelles nous avons pu, par rétro-analyse, estimer une valeur du coefficient de perméabilité isotrope équivalent.

Ces données êtaient les suivantes:

- dimensions de la fouille;

- fiche des parois moulées dans la molasse:

- hauteur de rabattement

- débit d'exhaure.

La rétro-analyse a utilisé deux méthodes

- la méthode simplifiée de Schneebelí (Schneebeli. 1987, cité par M. Cassan 1994)

- la méthode complète de Harr (Harr, 1962, cité par M. Cassan, 1994).

Les résultats sont donnés dans le tableau I, soit :

$$
1.9 .10^{-5} \leq \mathrm{k} \leq 4.6 .10^{-5} \mathrm{~m} / \mathrm{s}
$$

Nous avons donc conservé la valeur obterue à partir de nos essais :

$$
\mathrm{k}=3.10^{-5} \mathrm{~m} / \mathrm{s}
$$

\begin{tabular}{|c|c|c|}
\hline Chatıtier & \multicolumn{2}{|c|}{$\mathrm{k}$ an $\mathrm{mo} / \mathrm{s}$} \\
\hline $\begin{array}{l}\text { Pue Bonnel } \\
\text { Rue Vendöme }\end{array}$ & $\begin{array}{c}2,5.10^{-5} \text { a } 3,5.10^{-5} \\
4,6.10^{-5}\end{array}$ & $\begin{array}{c}1,9.10^{-5} \cdot 2,5.10^{-5} \\
3,7,10^{-5}\end{array}$ \\
\hline
\end{tabular}

TABLEAL III Évaluation de $\mathrm{k}$ par rétro-analyse de données de chantiers.
Nous avons alors évalué les débits de pompage de la foujlle du palais de fustice ả l'aide des deux néthodes uitilisées pour le rétro-analyse. Nous avons ainsi obtena

$$
410 \leq \mathrm{Q} \leq 585 \mathrm{~m}^{3} / \text { helre }
$$

soit une valeur moyemne:

$$
\mathrm{Q}=500 \mathrm{~m}^{3} / \text { heure }
$$

Le débit maximal pompé perdant le chantier a été :

$$
\mathrm{Q}=460 \mathrm{~m}^{3} / \text { heure }
$$

et le débit d"exploitation actuel est :

$$
\mathrm{Q}=360 \mathrm{~m}^{3 / \text { heure }}
$$

Ces résultats se passent de commentaires.

\section{4}

\section{Conclusion}

La méthode d'interprétation des essais Lefranc présentée et analysée dans cette commuizication ast utilisée depuis plusieurs années déjà et a été intếgrée à la nome NFP.94.132 dans une annexe non normative.

L'analyse détaillèe à laquelle nous avons procédé montre que cette méthode permet de valariser consjdérablement l'essai Lefranc et de Iimiter les controverses dont il continue de faire l'objet bien que celles-ci se sojent délà bien attếnués.

Cette méthode permet, en effet, de suive le dérou lement du phénomène de percolation de l'eà dans la cavité et de mettre en évidence les anomalies et les perturbations qui ont pu se produire pendant la durée de l'essaí. Comme nous l'avons vu sur des exemples réels, elle permet de dégager des valeurs du coefficient de perméabilité tout à fait représentatives à partir d'essais qui auraient été considérés, jadis, comme non interprétables et par conséquent éliminés, ou au contraire qui auraient été interprétês dans leur phase finale. Cette interprétation aurait donc intégré toutes les perturbations qui auraient pu se produire et aurait ainsi conduit a des résultats non représentatifs, avec toutes les conséquences fấcheuses et quelquefois même désastreuses que cela implique.

Mais il ne faut pas se leurrer, l'essai Lefranc a ses limites et, appliqué à des terrains de fajble perméabilité. il ne peut conduire qu'à des déboires. Rappelons 
cu“il n'est applicable qu'à des terrains dont Je coefficient de perméabilité est supérieur à $10^{-1 *} \mathrm{~m} / \mathrm{s}$.

Sa simplicité est, certes, très attrayante, mais elle est trompeuse car d'une part, la réalisation de la cavité de filtration est très délicate, et d'autre part la méthode par injection qui est pratiquement généralisẻe à l'heure actuelle peut entrainer, dans certains terrains, des colmatages immédiats et indétectables mêne avec la méthode d'interprétation présentée ici. On retiendra que cette méthode par injection est surtout valable dans les terrains granulaires, et la mêthode par pompage dans les terrains douês d'une légère cohésion

Lorsqu'on approche de la limite inférieure de validité de l'essai, c'est-商-dire lorsqu'on se trouve dans des terrains â forte prédominance argíleuse ou limoneuse on peut, soit vider le forage (pompe ou émulsenr) soit au contraire le remplir et, aprés arrêt de la pompe, étudier l'évolution de la charge par la méthode du débit nut.

Mais surtout il ne faut pas perdre de vue que l'essai Lefranc n'est qu'un essai ponctuel et qu'il est très dangereux de vouloir extrapoler sans précaution. ses résultats à l'ensemble d'un site, mème lorsqu'on en a réalisé un grand nombre. Il serait donc inadmissible de se limiter a de simples essais Lefranc pour étudier un rabattement de nappe important. En revanche, ils donnent une idée très représentative des contrastes de perméabilité et, à ce titre, ils peuvent être un excellent auxiliaire des essais de pompage et devraient toujours précéder et accompagner ces essajs par exemple en profitart des forages des pié. zomètres satellites.

La normalisation des essais d'ea u doit, certes, permettre d’améliorer la qualité de l'expérimentation et les progrès réalisés dans le matêrriel et les méthodes d'interprétation doivent accroître la fiabilité des résultats, mais il est bien évident que les coefícients de perméabilité ainsi déterminés n'ont. conme la plupart des paramètres géotechniques, qu'un caractère conventionnel. Il ne faut donc pas attencre des miracles de ces normalisations car la nature est, et restera capricieuse et mystérieuse et c'est à la sagacité mais aussía à la rigueur de l'ingênieur qu'll appartiendra finalement de percer ses mysteres et de composer avec ses caprices.

\section{Bibliographie}

Boulton N.S. - Untersdy radial flow to a punped well allowing for delayed yield from storage. AlHS Assemblé gênerale de Rome, Tome II, publication $n^{\circ} 37,1954$.

Boulton N.S - Amalysis of data from non écuilibrium pumping test atlowing from delayed yieds. Proc. Inst. Civ. Eng. vol. 26, nov, 1963.

Brillant J. - Appareil pour la mesure de la perméabilitẻ du terrah en place. Génie chiา aoủt 1960 .
Britant J. - La mesure in situ des permegbilités locales. Geotechnique, Londres, miar's 1966.

Cassan M. - Aide-mémoire d'hydraulique souterraine. Presses des ponts et chaussters, 1994.

Cassan M. - Les essais de perméabilité sur site dans la reconnaissance des sols. Presses des ponts et chaussées, 2004. $2^{\circ}$ éd.

Haniush M.S. - Non steady radial flow in an infinite leaky aguifer. Transaction
American Geophysical Unido, vol, 36 $n^{\circ} 1$, févitier 1955 .

Hanush MS - Hydraulic of wells Advence Hydroscience, Tome I, Ven Te Chow. New York, 1964.

Harr M.E. - Groundwater and scepage. Mac Graw Hill Book Company, New York, 1962

Schneebel G. - Hydraulique souteraine. Eyroiles, 1987.

Theïs C.V. - The Source of Water Derived from Wells. Civit Eñ vol. 10. 1940. 